\title{
Motion Reliability Analysis of Unlocking Trigger Device Based on CPSO-BR-BP Neural Network with Uncertain Parameters
}

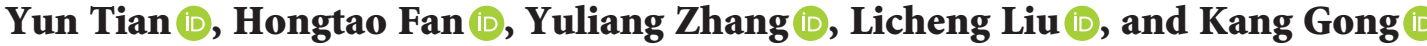 \\ Beijing Spacecrafts, Beijing 100083, China \\ Correspondence should be addressed to Yuliang Zhang; 1317906366@qq.com
}

Received 8 July 2021; Accepted 13 August 2021; Published 21 August 2021

Academic Editor: Mohammad Yazdi

Copyright (c) 2021 Yun Tian et al. This is an open access article distributed under the Creative Commons Attribution License, which permits unrestricted use, distribution, and reproduction in any medium, provided the original work is properly cited.

\begin{abstract}
Aiming at overcoming the problem that the mechanism function of the unlocking trigger device is difficult to obtain and the corresponding reliability analysis cannot be performed, a motion reliability analysis method based on the CPSO-BR-BP neural network proxy model is proposed. Firstly, the particle swarm algorithm is optimized through the chaotic sequence, and the backpropagation (BP) neural network is optimized using Chaos Particle Swarm Optimization (CPSO) and Bayesian Regularization (BR) algorithm. The CPSO-BR-BP neural network proxy model is established, and the reliability of shape memory alloys (SMA) wire unlocking based on the structural function is calculated. Moreover, according to the structural function of the separation process, the motion reliability based on the proxy model and the improved membership function is calculated. Finally, a series reliability model is established based on the unlocking process and the separation process to calculate the reliability of the whole machine. The reliability of the unlocking trigger device is analyzed by the proposed method. Results show that the proposed method is computationally efficient with the calculated reliability of 0.9987 .
\end{abstract}

\section{Introduction}

The connection and separation device in the spacecraft is designed to realize the connection between spacecraft sections as well as that between the body and components in the launch section. At the same time, it achieves reliable unlocking and separation on the orbit according to the established requirements. The reliability can not only quantify the motion performance of the device under the condition of parameter uncertainty but also provide the necessary theoretical basis for its further optimization [1-5]. Future space station construction and manned missions to the moon make higher demands on the reliability, safety, and separation impact of the connection and separation device, and also more urgent needs for the connection and separation module with large load capacity and low impact are put forward $[6,7]$.

To reduce the separation impact of the device, ensure the safety of connection and separation, and avoid space pollution, researchers have applied shape memory alloys (SMA) to achieve the unlocking function [8-13]. Zhang et al. [8] designed a separation release device using SMA wire. The device has a simple structure, but it lacks further tests on impact performance and load-bearing capacity. Hu et al. [9] proposed an unlocking mechanism based on memory alloy drive with non-self-locking transmission characteristics and multistage force-increasing structure. The key parameters affecting the drive performance of memory alloy and the design of non-self-locking trapezoidal thread pairs were determined through simulation, but the impact of parameter uncertainty on device performance was not considered. Han et al. [10] proposed a low-impact, light-weight, interlocking connection and separation device based on the shape memory alloy tube unlocking. The key parameters were determined through theoretical analysis and simulation, and the unlocking impact and unlocking time of this prototype were tested. However, the reliability analysis of the device was not performed. Most of the above-mentioned studies were carried out on deterministic experiments or simulations to determine the structural parameters of the device, without considering the influences of parameter uncertainties on its motion reliability. Reliability analysis is the focus of the design process, which can be used to conduct quantitative analysis of sports 
performance under deterministic conditions. Due to the complex structure of the unlocking trigger device, it is difficult to directly obtain its structure function. The proxy model is introduced to establish the mapping relationships between the uncertainty parameters and the responses.

Currently, the most widely used proxy models are polynomial response surface proxy model [11], Kriging proxy model [12-14], radial basis function proxy model $[15,16]$, and BP neural network proxy model [17-19]. Among them, the BP neural network proxy model significantly improves the robustness of the overall design of the mechanical structure with low calculation cost and high noise processing capability. However, the gradient descent method used by BP neural network models often converges to a local optimal solution, which affects the accuracy and efficiency of proxy models. For this reason, researchers have optimized the BP neural network models [20-23]. Tang et al. [20] proposed an analytic method for the reliability and sensitivity of motion mechanisms based on BP neural networks, which can effectively improve the reliability and robustness of machine tool motion mechanisms. Dai et al. [21] proposed an explorer optimization algorithm based on heuristic search, which trains the neural network through evolutionary methods to adjust the structure and parameters of the neural network. Gong et al. [22] improved the particle swarm optimization (PSO) by evolutionary strategy to optimize a BP neural network for storage reliability prediction and combined the global search capability of PSO with the local search capability of the BP network to improve the convergence speed and prediction accuracy of the algorithm. Yan et al. [23] proposed an artificial neural network model based on genetic algorithm optimization to analyze the reliability of aviation bearing fatigue and overcomed the problem of artificial neural network local optimization and premature convergence problems. The above studies mostly optimize the characteristics of BP neural network models and do not analyze the accuracy and efficiency of proxy models. Moreover, they are difficult to solve the reliability issues of actual engineering cases.

To this end, considering working principle of unlocking trigger device and uncertain parameters during movement, a new reliability analysis method based on CPSO-BR-BP neural network (Chaos Particle Swarm OptimizationBayesian Regularization-BP neural network) is proposed, and the unlocking trigger device is used as the research object. The influence of each uncertainty parameter on the device performance is clarified, and the motion reliability of the SMA wire unlocking and separation process is analyzed under different coefficients to verify its motion reliability under uncertain parameters. The research method provides a reliable theoretical reference for further improving the structural performance of the unlocking trigger device.

\section{Configuration and Working Principle of Unlocking Trigger Device}

The overall configuration of unlocking trigger device is shown in Figure 1. The device is mainly composed of three parts: the SMA wire trigger component, the unlocking and transmission component, and the connection and separation component. Among them, the SMA wire trigger component plays a reliable limit and lock function when locked, and it provides trigger driving force for releasing the limit and lock when unlocked. The connection and separation component can realize the loading of the pretightening force to keep overall connection strength and rigidity when locked. Besides, it can quickly realize the separation of the connecting parts while unlocking. The unlocking and transmission components can complete the load amplification and reduce the impact while unlocking [6].

The unlocking trigger device is a new type of nonpyrotechnic unlocking device. It is to make the nut of the non-self-locking thread pair into a flywheel. When the connection is locked, the flywheel nut is restricted by the triggering mechanism to rotate in the circumferential direction. Besides, it is combined with the non-self-locking thread to realize the connection of the spacecraft and its accessories. To ensure the tightness of the connection, the lower end of the screw is pretightened by the loading nut to provide the unlocking driving force. At this time, the flywheel nut is in the unlocked state under the action of nonself-locking force. When it is released, the SMA wire is heated by electricity to shrink it to release the restriction of the locking mechanism on the nut. The flywheel nut is unlocked by inertial reversal under the action of the nonself-locking force of the threaded connection. The device can not only complete the heavy-duty connection through the thread pair connection but also complete the separation of the screw and the nut by releasing the non-self-locking thread after the nut limit to achieve the goal of low-impact separation. The working principle is shown in Figure 2.

\section{Reliability Analysis Method Based on CPSO- BR-BP Neural Network Proxy Model}

The premise of the reliability analysis of the unlocking trigger device is to construct the functional function of the mechanism. Since it is difficult to obtain the physical equation of the device uncertainty parameter and its response, the key is to obtain the relationship between the uncertainty parameter and its response, that is, the proxy model. Currently, the most widely used proxy models are polynomial response surface proxy model [11], Kriging proxy model [12-14], radial basis function proxy model $[15,16]$, and BP neural network proxy model [17-19]. Among them, the BP neural network proxy model significantly improved the robustness of the overall design of the mechanical structure with its low calculation cost and high noise processing capability [17]. To improve the accuracy and efficiency of the reliability calculation, the motion reliability analysis of the SMA wire unlocking process and the separation process is carried out based on the BP neural network proxy model.

3.1. BP Neural Network Proxy Model. BP neural network is an intelligent algorithm with error back-propagation. Its topological structure is mainly composed of three parts: 


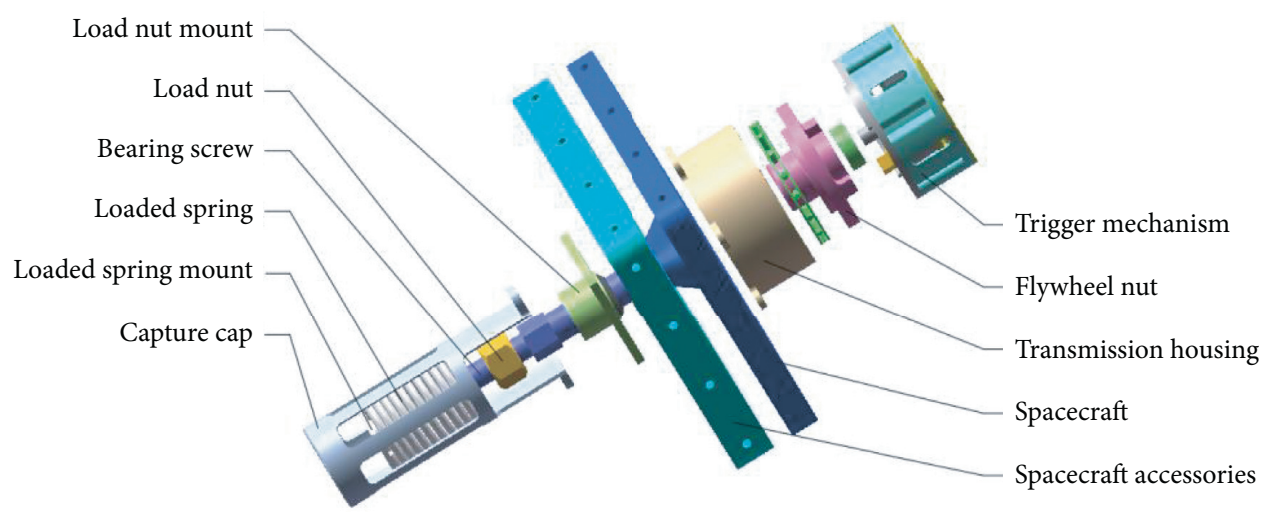

FIGURE 1: The overall configuration diagram of the unlocking trigger device.

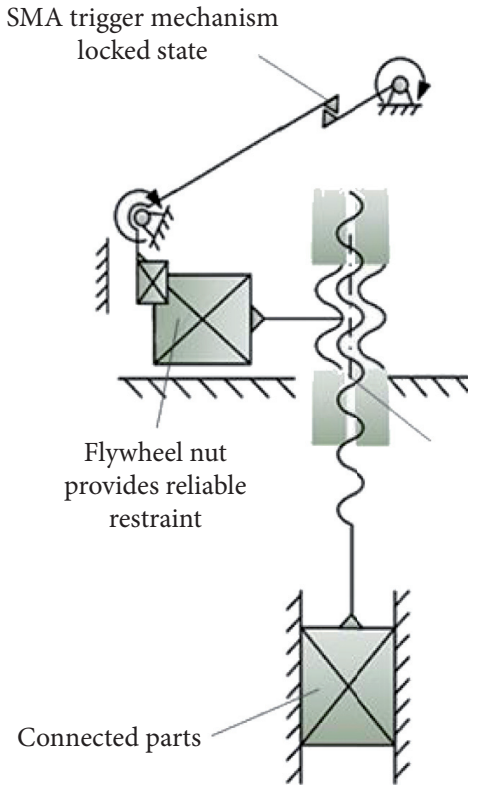

(a)

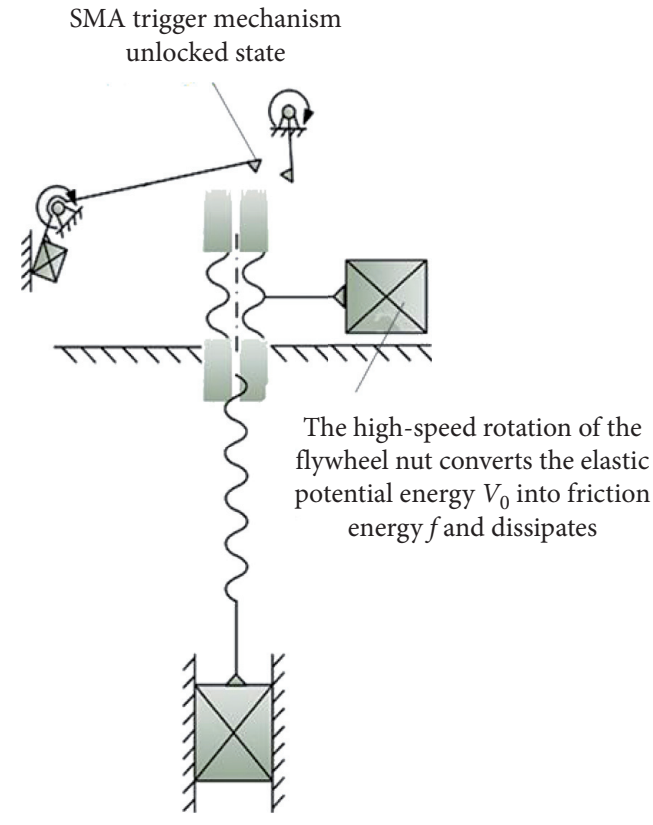

(b)

FIGURE 2: Working principle diagram of the separation device: (a) the connection status and (b) the separation status.

input layer, hidden layer, and output layer. Each layer is connected by neurons. Assuming that the nodes of the input layer, hidden layer, and output layer in the three-layer structure are $m, n$, and $r$, respectively, the function transmitted by the neuron can be expressed by

$$
\begin{aligned}
& h_{k}=f_{1}\left(\sum_{i=1}^{m} W_{i k} x_{i}+b_{k}\right), \quad(j=1,2, \ldots, r),(i=1,2, \ldots, m),(k=1,2, \ldots, n), \\
& y_{k}=f_{2}\left(\sum_{k=1}^{n} W_{k j} h_{k}+b_{j}\right), \quad(j=1,2, \ldots, r),(i=1,2, \ldots, m),(k=1,2, \ldots, n),
\end{aligned}
$$

where $h_{k}$ is the output of the hidden layer; $y_{k}$ represents the output of the output layer; $f_{1}(x)$ and $f_{2}(x)$ are the activation functions; $x_{i}$ denotes the input variable; $b_{k}$ is the threshold of the hidden layer; $b_{j}$ is the threshold of the output layer; $W_{i k}$ reflects the weight between the hidden layer and the input layer; and $W_{k j}$ is the weight between the output layer and the hidden layer. 
According to equations (1) and (2), the mapping relationship between the network input variable $x_{i}$ and the output response $y$ can be expressed as

$$
y=f_{2}\left(\sum_{k=1}^{n} W_{j k} f_{1}\left(\sum_{i=1}^{m} W_{i k} x_{i}+b_{k}\right)+b_{j}\right) .
$$

The BP neural network uses the gradient descent method as the training algorithm and the training error of the network as the performance function. The weights and thresholds in equation (3) can be iterated to make the fitting accuracy of the BP neural network proxy model reach the allowable error range. The performance function characterized by the gradient descent method can be expressed as

$$
E_{D}=\sum_{i=1}^{s}\left(T_{i}-y_{i}\right)^{2}
$$

where $s$ is the total number of weights; $T_{i}$ is the actual output; and $y_{i}$ is the network prediction output.

However, the gradient descent method often converges to the local optimal solution in which the initial values of weights and thresholds are randomly generated in the training process. This makes the network not fully trained, which will affect the fitting accuracy and computational efficiency of the agent model. Therefore, the Chaos Particle Swarm Optimization (CPSO) and Bayesian Regularization (BR) algorithm are used to optimize the BP neural network to improve the calculation efficiency as well as ensuring the calculation accuracy of the proxy model.

3.2. Chaos Particle Swarm Optimization. The particle swarm algorithm is an optimization algorithm developed based on the actual biological group activities. The significant features of the algorithm are fast convergence, high robustness, and strong global optimization ability. The particle swarm algorithm is prone to random oscillations and falls into local optimal values. To solve this problem, chaos algorithm is introduced into the particle swarm algorithm. In addition, the chaotic sequence is used to iterate the optimal particles in the particle swarm to improve the ability of the algorithm to jump out of the local optimal solution. The specific steps are given as follows:

(1) Initialize the population. Then, determine the population number $N$, dimension $D$, the maximum number of iterations $K_{\max }$, and other parameters.

(2) Calculate the fitness of each particle and update the local optimal position $P_{\text {best }}$ and the global optimal position $g_{\text {best }}$ of each particle.

(3) Normalize the optimal position of each particle $P_{\text {best }}=\left(P_{g 1}, P_{g 2}, \ldots, P_{g D}\right)$ to obtain $\left(y_{k 1}, P_{k 2}, \ldots\right.$, $\left.y_{k D}\right)$.

(4) Use the logistic equation to iterate on $y_{k 1}$ and denormalize the obtained results.

(5) Use the obtained new solution to calculate the fitness. If the new solution is better than the old solution, output the new one.
(6) Judge whether the maximum number of chaotic iterations is reached; if not, return to step (2).

\subsection{CPSO-BR-BP Neural Network Proxy Model.}

According to the advantages of CPSO and BR algorithms in correcting weights and thresholds, the traditional BP neural network is optimized and a new CPSO-BR-BP neural network proxy model is proposed. The basic idea is as follows. Firstly, set the basic parameters of the chaotic particle swarm, determine the number of random variables and responses, randomly generate the initial position and initial velocity of the particle, update the particle velocity and position, and get the individual extreme value and global extreme value of the particle by using the mean square error of the BP neural network as the fitness function. Secondly, the global extreme value of the particle is optimized chaotically by the logistic equation. The best individual is output and used as the optimal initial weight and threshold. Finally, the $\mathrm{BP}$ neural network that obtains the optimal initial value is used for training by $\mathrm{BR}$, and the correction function is introduced based on equation (4) to optimize the performance function. The revised performance function expression is as follows:

$$
\begin{aligned}
E & =\alpha E_{D}+\beta E_{W}, \\
E_{w} & =\frac{1}{s} \sum_{i=1}^{s} w_{i}^{2},
\end{aligned}
$$

where $E$ is the modified performance function; $\alpha$ and $\beta$ are the regularization coefficients; $E_{w}$ is the weight attenuation term; and $\omega_{i}$ is the weight of the neural network connection.

The BR algorithm that takes equation (5) as a performance function adaptively adjusts the size of $\alpha$ and $\beta$ during the training process. Under the condition of ensuring that the training error converges to the target error, the final weight and threshold are output, and CPSO-BR-BP neural network proxy model is established based on this.

The main process of the reliability analysis of the unlocking trigger device based on the CPSO-BR-BP neural network under the parameter uncertainty is shown in Figure 3 .

\subsection{Reliability Calculation Method Based on Improved Membership}

3.4.1. Reliability Calculation Method of SMA Wire Unlocking Process. The successful completion of the unlocking process of the device within the specified time is the basis for ensuring its reliability. The mapping relationship between the uncertain parameters and the response is constructed, which is based on the BP neural network, and the key performance indicator is the recovery displacement. The probability distribution characteristics of the recovery displacement of the SMA wire under the condition of parameter uncertainty are obtained by the mapping relationship. The unlocking reliability of the shape memory alloy wire is obtained based on the allowable displacement. The function expression of 


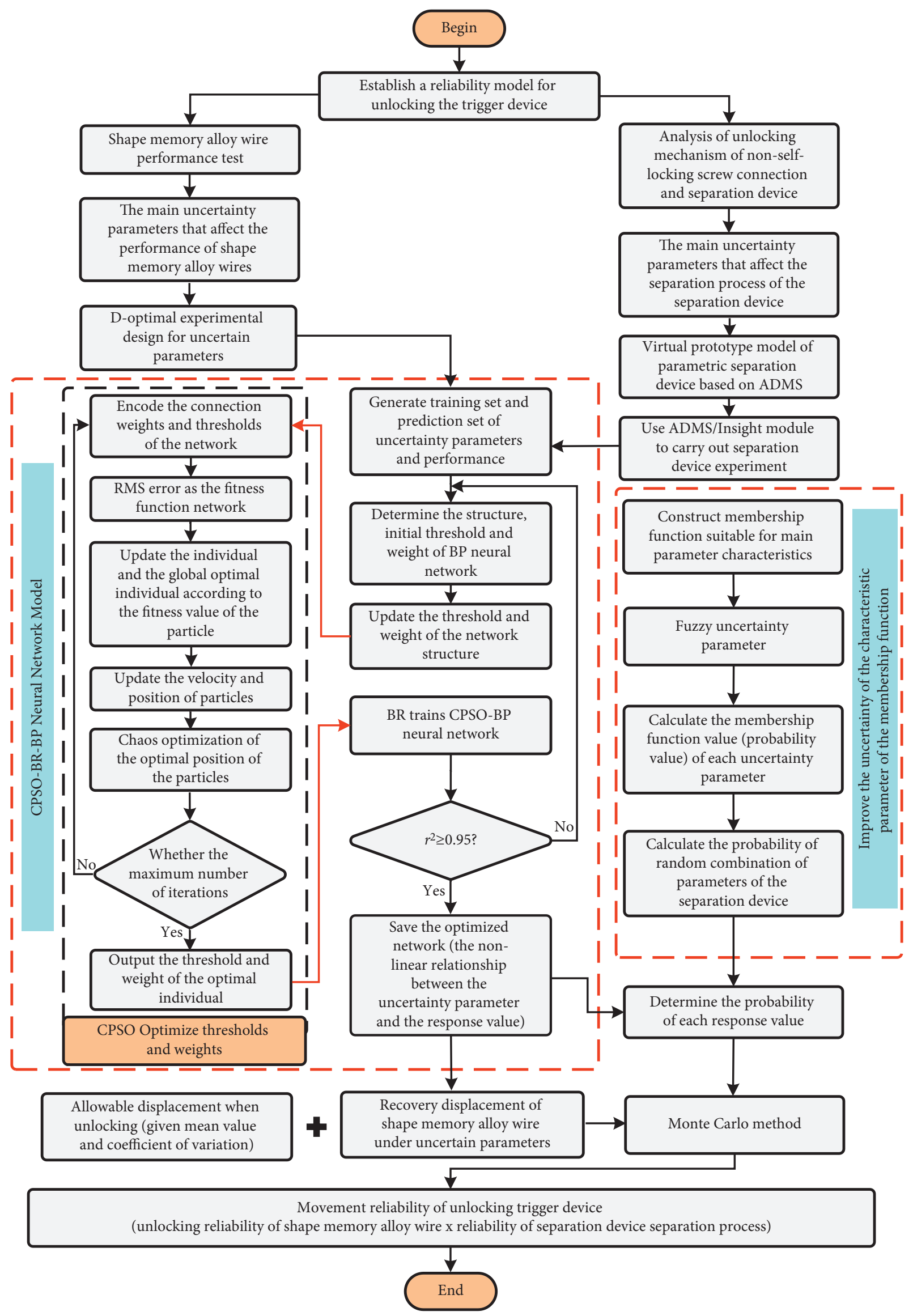

FIGURE 3: Main processes of reliability analysis of separation mechanism. 
the SMA wire unlocking reliability analysis function can be represented as

$$
X=L(x)-L=\left\{\begin{array}{cl}
>0, & \text { displacement is greater than allowable displacement } \\
=0, & \text { displacement is equal to allowable displacement } \\
<0, & \text { displacement is less than allowable displacement }
\end{array}\right.
$$

where $L(x)$ is the return displacement of the SMA wire; $L$ is the allowable displacement when the SMA wire is unlocked.

According to equation (7), the reliability can be defined as

$$
R(t)=\frac{n}{N}
$$

\subsubsection{Reliability Calculation Method of the Separation} Process. In the process of calculating motion reliability, a reasonable membership function is a prerequisite for the quantification of parameter uncertainty. The traditional distributions are in the neighborhood centered on the fuzzy median. It is necessary to artificially determine the value interval of the uncertainty parameter. Besides, it is difficult to reflect the actual value of the parameter. Considering the actual mechanical structure, the uncertainty parameters are mostly in the $6 \sigma$ neighborhood centered on its mean value, and the reliability of mechanical parts based on this range can meet the actual engineering requirement [24]. The membership function based on the $6 \sigma$ principle is improved to reduce the deviation between the uncertainty parameter and the actual manufacturing, and it also can improve the accuracy of the motion reliability analysis of the separation process.

The improved membership function can be represented as

$$
A\left(x_{i}\right)= \begin{cases}0, & x_{i} \leq \mu_{i}-6 \sigma_{i}, \\ \sqrt[d]{\frac{x_{i}-\mu_{i}+6 \sigma_{i}}{6 \sigma_{i}},} & \mu_{i}-6 \sigma_{i}<x_{i} \leq \mu_{i}, \\ \sqrt[d]{\frac{-x_{i}+\mu_{i}+6 \sigma_{i}}{6 \sigma_{i}},} & \mu_{i}<x_{i} \leq \mu_{i}+6 \sigma_{i}, \\ 0, & \mu_{i}+6 \sigma_{i} \leq x_{i},\end{cases}
$$

where $x_{i}$ is the value of a random variable; $A\left(x_{i}\right)$ is the degree of membership corresponding to the random variable $x_{i} ; \mu_{i}$ is the mean value of the random variable $x_{i} ; \sigma_{i}$ is the standard deviation of the random variable $x_{i}$; $d$ is the undetermined coefficient.

The smooth completion of the separation process of the device within the specified time is the basis and prerequisite to ensure its movement reliability. For this reason, the fuzzy reliability model of the separation device is established, and the key performance index is the separation time of the device. Therefore, the separation process of the separation device can be calculated. According to the overall separation time index of the unlocking trigger device, the threshold of the response time of the separation process is determined, and the movement reliability function is constructed. The function can be represented as

$$
G\left(x_{i}\right)=0.0804-t_{2}\left(x_{i}\right)= \begin{cases}>0, & \text { reliable separation process, } \\ =0, & \text { limit state separation process, } \\ <0, & \text { failure of the separation process }\end{cases}
$$

where $t_{2}\left(x_{i}\right)$ is the response value under each uncertainty parameter combination, $s ; x_{i}$ is a set of uncertainty design parameters selected at random.

According to equation (10), the probability of each uncertainty parameter combination can be calculated, and its expression is as follows:

$$
p\left(G\left(x_{j}\right)\right)=\prod_{i=1}^{n} A\left(x_{i}\right)
$$

where $i$ is the number of uncertain parameters considered, $i=1,2, \ldots, n ; j$ is the number of Monte Carlo simulations, $j=1,2, \ldots, m$.

According to equation (11), the calculation formula of the movement reliability of the separation process can be expressed as

$$
\begin{aligned}
R(G(x)) & =\frac{\sum_{j=1}^{m} P\left(G\left(x_{j}\right)\right)}{m}, \\
P\left(G\left(x_{j}\right)\right) & = \begin{cases}p\left(G\left(x_{j}\right)\right), & 0 \leq G\left(x_{j}\right), \\
1-p\left(G\left(x_{j}\right)\right), & 0>G\left(x_{j}\right) .\end{cases}
\end{aligned}
$$

\subsubsection{Reliability Calculation Method of Unlocking Trigger} Device. The unlocking trigger device consists of the SMA wire unlocking process and the separation device separation process according to the working principle, and the movement process conforms to the characteristics of the series reliability model. This means that any part of the failure will cause the entire system to fail during the movement of the unlocking trigger device. Its reliability block diagram is shown in Figure 4.

The reliability of the series system is equal to the product of the reliability of each subsystem, and its expression can be described as follows: 


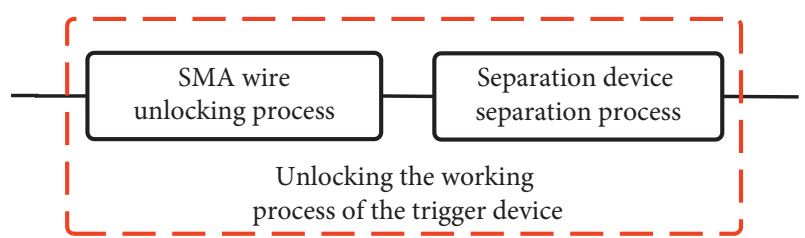

FIgURE 4: Series reliability model of the separation mechanism.

$$
R(t)=\prod_{i=1}^{n} R_{i}(t),
$$

where $R(t)$ is the reliability of the system, that is, the reliability of the whole machine for unlocking the trigger device; $R_{i}(t)$ is the reliability of each unit, that is, the reliability of the SMA wire unlocking or the separation device separation process.

\section{Reliability Analysis of the Unlocking Trigger Device}

The unlocking trigger device is effectively and reliably locked during the launch phase. After reaching the predetermined orbit, the unlocking action needs to be completed through the SMA wire, and the separation of the carrier and the payload is accomplished. In the process of analyzing the movement reliability of the whole machine, firstly, the movement reliability of the SMA wire in unlocking is analyzed. Then the movement reliability of the mechanical separation process is verified to obtain the reliability of the separation time under the fluctuation of the uncertainty parameter. Finally, the reliability of the whole machine is analyzed.

\subsection{Analysis of Motion Reliability of SMA Wire in Unlocking}

4.1.1. Reliability Calculation Method of Unlocking Trigger Device. Through the performance test of the SMA wire under different parameter conditions, the current, the time, the load, the wire diameter, and the temperature have significant impacts on the performance of the SMA wire, and there is large uncertainty in the working process. Therefore, the parameters are the main parameters that affect the reliability of its motion. The traditional performance analysis is carried out by changing a single parameter without efficient experimental design plans. For this reason, the D-optimal experimental design is used for the experimental design [25], and the CPSO-BR-BP neural network proxy model is used to obtain the relationship between inputs and outputs. Then the continuous characterization of the uncertainty parameter and the performance of the SMA wire can be set up, and the influences of parameter uncertainty on the performance of the SMA wire can be shown. According to the main parameters of traditional SMA wire performance analysis and the field test environment, the statistical values of each parameter are shown in Table 1, and the experimental design scheme and response values are shown in Table 2.

To observe the effects of different random parameters on the recovery time and recovery displacement of SMA wire more clearly, according to the test data and results in Table 2, the sample space of recovery time and recovery displacement under the action of any two uncertain parameters are shown in Figure 5.

It can be seen from Figure 5 that the recovery time and the recovery displacement of the SMA wire are evenly distributed in the sample space under the action of different random parameters with a large dispersion. This indicates that the sample value obtained through the D-optimal experimental design can reflect the variation range of the response value of random parameters. The proxy model established based on this set of data can more accurately characterize the mapping relationship between inputs and outputs.

25 sets of data from the sample data in Table 2 are randomly extracted for normalization, which are used as the prediction data of the neural network agent model. Besides, the remaining 6 sets of data are used as test data to test the prediction accuracy of the neural network proxy model. The proxy model of the uncertainty parameters on the response time of the SMA wire is obtained by predicting. The proxy model can be shown as

$$
\begin{aligned}
t_{1}= & \frac{0.6497}{A_{1}}-\frac{2.2306}{A_{2}}+\frac{0.7450}{A_{3}}+\frac{0.0059}{A_{4}}+\frac{0.4022}{A_{5}} \\
& +\frac{0.3936}{A_{6}}+\frac{0.3936}{A_{7}}-0.2406,
\end{aligned}
$$

where

$$
\begin{aligned}
A_{1}= & 1+\exp \left(0.2852 I+0.1185 t^{\prime}+0.0054 d\right. \\
& +0.3902 F-0.5300 T+0.8283), \\
A_{2}= & 1+\exp \left(0.5403 I-0.0716 t^{\prime}-1.6457 d\right. \\
& -0.0925 F+1.5852 T+2.7776), \\
A_{3}= & 1+\exp \left(0.3202 I+0.2112 t^{\prime}-0.0746 d\right. \\
& +0.5373 F-0.5014 T+0.9512), \\
A_{4}= & 1+\exp \left(-0.2345 I-0.2453 t^{\prime}+0.0687 d\right. \\
& -0.2266 F+0.0678 T+0.1210), \\
A_{5}= & 1+\exp \left(-0.4772 I-0.2026 t^{\prime}+0.1036 d\right. \\
& -0.5309 F+0.0928 T+0.3704), \\
A_{6}= & 1+\exp \left(-0.4741 I-0.2099 t^{\prime}+0.1063 d\right. \\
& -0.5222 F+0.0962 T+0.3668), \\
A_{7}= & 1+\exp \left(-0.4417 I-0.2242 t^{\prime}+0.1008 d\right. \\
& -0.4833 F+0.0914 T+0.3371) .
\end{aligned}
$$

The proxy model of uncertainty parameters on the recovery displacement of SMA wire is shown as

$$
\begin{aligned}
l= & \frac{2.6947}{B_{1}}-\frac{0.3673}{B_{2}}-\frac{1.0795}{B_{3}}-\frac{0.5471}{B_{4}} \\
& -\frac{0.6164}{B_{5}}+\frac{0.2473}{B_{6}}-\frac{0.4259}{B_{7}}+0.1161,
\end{aligned}
$$


TABLE 1: Statistical values of uncertain parameters.

\begin{tabular}{|c|c|c|c|c|c|}
\hline Uncertainty parameter & Symbol & Unit & Lower bound & Mean & Upper bound \\
\hline Heating current & $I$ & $\mathrm{~A}$ & 4.50 & 5.00 & 5.50 \\
\hline Heating time & $t \prime$ & $\mathrm{ms}$ & 90.00 & 100.00 & 110.00 \\
\hline Wire diameter & $d$ & $\mathrm{~mm}$ & 0.25 & 0.30 & 0.35 \\
\hline Load & $F$ & $\mathrm{~N}$ & 7.00 & 8.00 & 9.00 \\
\hline Temperature & $T$ & ${ }^{\circ} \mathrm{C}$ & -70.00 & 0 & 70.00 \\
\hline
\end{tabular}

TABLE 2: D-optimal experimental design and response values.

\begin{tabular}{|c|c|c|c|c|c|c|c|}
\hline No. & Heating current & Heating time & Wire diameter & Load & Temperature & Response time & Response displacement \\
\hline 1 & 5.50 & 90.0 & 0.25 & 7.00 & 70.0 & 4.393 & 11.172 \\
\hline 2 & 5.07 & 110.0 & 0.25 & 9.00 & 70.0 & 6.058 & 11.628 \\
\hline 3 & 4.50 & 110.0 & 0.35 & 7.00 & -70.0 & 140.306 & 11.989 \\
\hline$\ldots$ & .. & $\ldots$ & $\ldots$ & . . & $\ldots$ & $\ldots$ & $\ldots$ \\
\hline 29 & 5.50 & 110.0 & 0.35 & 7.00 & 70.0 & 11.932 & 7.695 \\
\hline 30 & 5.07 & 110.0 & 0.25 & 9.00 & 70.0 & 6.058 & 11.628 \\
\hline 31 & 5.50 & 101.6 & 0.25 & 8.15 & 70.0 & 4.826 & 11.609 \\
\hline
\end{tabular}

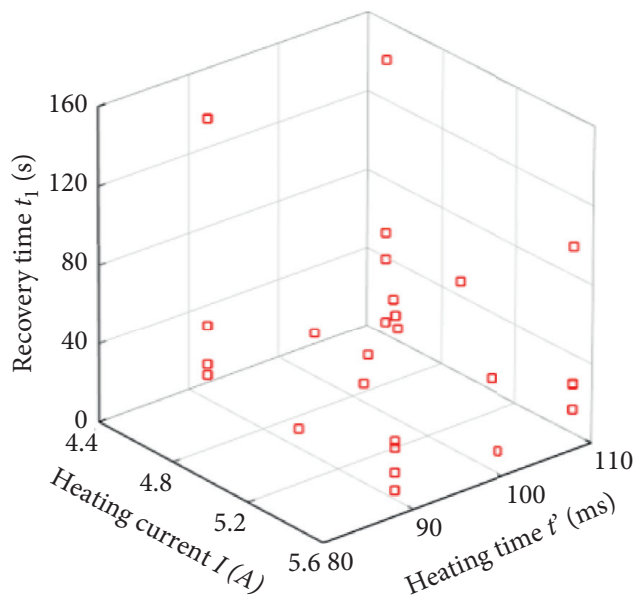

(a)

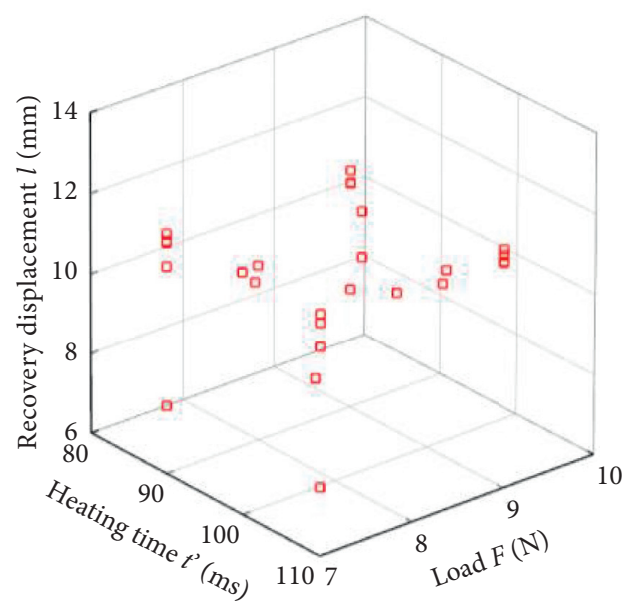

(c)

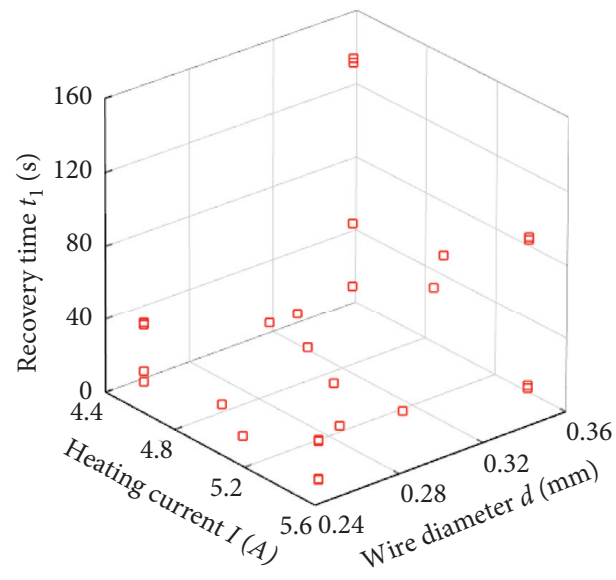

(b)

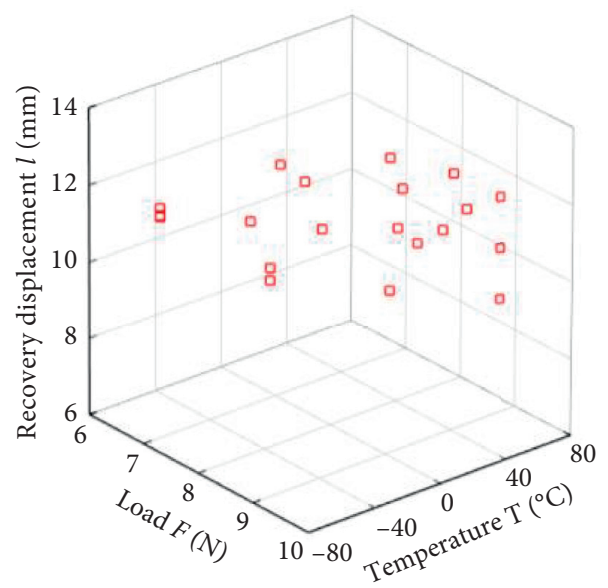

(d)

Figure 5: Sample space of uncertain parameters for heating current and heating time (a), heating current and wire diameter (b), heating time and load (c), and load and temperature (d). 
where

$$
\begin{aligned}
B_{1}= & 1+\exp \left(0.5639 I+0.8471 t^{\prime}-1.4172 d\right. \\
& +0.7140 F-4.1718 T+4.1867), \\
B_{2}= & 1+\exp \left(-0.1706-0.0847 t^{\prime}+0.0924 d\right. \\
& +0.2513 F+0.3391 T+0.5180), \\
B_{3}= & +\exp \left(0.4742 I+0.2539 t^{\prime}-0.7967 d\right. \\
& +0.4270 F-2.0559 T+0.9893), \\
B_{4}= & +\exp \left(-0.3780 I+0.1858 t^{\prime}-0.1279 d\right. \\
& +0.2607 F+0.55330 T+0.2446), \\
B_{5}= & +\exp \left(-0.4602 I-0.2625 t^{\prime}-0.3707 d\right. \\
& -0.8604 F-0.4454 T+0.6977), \\
B_{6}= & 1+\exp \left(0.0307 I-0.0019 t^{\prime}-0.1778 d\right. \\
& -0.0256 F-0.0648 T+0.1118), \\
B_{7}= & 1+\exp \left(-0.1985 I-0.0520 t^{\prime}+0.0631 d\right. \\
& +0.2778 F+0.3952 T+0.2028) .
\end{aligned}
$$

The measurement coefficient is used to evaluate the accuracy of the CPSO-BR-BP proxy model so that the established proxy model has high accuracy to predict the response of the actual SMA wire under uncertainty parameters. The response time and the response displacement are tested on the accuracy of the proxy model, respectively. The results are shown in Table 3.

It can be seen from Table 3 that the determination coefficient values of the CPSO-BR-BP proxy model for the response time and the response displacement are both close to 1 . This indicates that the predicted data obtained from the proxy model is relatively close to the actual values. Besides, the established proxy model can reflect the changing trend and specific values of the response value of the SMA wire with high accuracy. To show the fitting accuracy of the proxy model more intuitively, the actual values of the test data are compared with the predicted values obtained from the CPSO-BR-BP proxy model. The corresponding change trends and prediction errors are shown in Figures 6 and 7 .

It can be seen from Figure 6 that the predicted response time and recovery displacement using the CPSO-BR-BP neural network proxy model are consistent with the actual value change trend, while the changing trend obtained from the traditional BP neural network is quite different. This indicates that the model can more accurately characterize the mapping relationship between the uncertainty parameters on the recovery time and the recovery displacement. In addition, to further verify the fitting accuracy of the proxy model, the error between the predicted values and the actual values concerning the two proxy models is compared. Results show that the error of the CPSO-BR-BP neural network proxy model presents a relatively stable fluctuation and all distributes around 0 , while the traditional BP neural network error is relatively larger with poorly stable fluctuations. This explains that the fitting accuracy of the improved proxy model is high, and it can accurately predict the recovery performance of the SMA wire under the condition of parameter uncertainty.
TABle 3: Accuracy verification results of the surrogate model.

\begin{tabular}{lc}
\hline Proxy model & Decisive factor $r^{2}$ \\
\hline Response time $t_{1}$ & 0.9915 \\
Response displacement $l$ & 0.9998 \\
\hline
\end{tabular}

4.1.2. Analysis of Motion Reliability of SMA Wire in Unlocking. According to the working principle of SMA wire and its role in the separation device, its reliability is defined as the ability to release the limit stably under the specified time and conditions. It is more important to achieve the limit release of the mechanism under specified conditions for SMA wire. Therefore, the limit state equation of SMA wire in unlocking is constructed based on the recovery displacement value and the design recovery displacement index of the SMA wire under the uncertainty parameters. The Monte Carlo method is used to analyze the motion reliability of SMA wire in unlocking, and its reliability value with a certain probability distribution is obtained. The uncertainty parameters and their response values obtained by Monte Carlo simulation are shown in Table 4 . The probability distribution characteristics of the uncertainty parameters are shown in Figures 8(a) to $8(\mathrm{e})$. The recovery displacement distribution of the SMA wire under the influence of the uncertainty parameters features is shown in Figure 8(f).

It can be seen from Figure $8(\mathrm{f})$ that the recovery displacement of the SMA wire shows a certain degree of fluctuation under the action of various uncertain parameters, and its value is mainly concentrated at about $12 \mathrm{~mm}$. In addition, the probability distribution characteristics approximately obey the normal distribution. Through the analysis of its truncated data, the recovery displacement of the SMA wire is lower than the design recovery displacement due to the influence of uncertainty parameters. This indicates that the uncertain parameters have effects on the SMA, such as heating current and heating time, which will reduce the reliability of unlocking. Compared with the deterministic SMA wire unlocking analysis in the traditional sense, the uncertainty analysis can better evaluate the reliability of the SMA wire unlocking under the action of uncertain parameters. Based on the reliability theory and Figure $8(f)$, the cumulative probability of the limit state function of the SMA wire is obtained, as shown in Figure 9.

It can be seen from Figure 9 that when the limit state function is smaller than 0 (i.e., the recovery displacement of the SMA wire fails to reach the specified value), the Monte Carlo method is used to obtain the failure probability of the SMA wire unlocking motion; that is, the reliability of the recovery displacement is 0.9996 . From the results of the reliability calculation, the uncertainty parameters have obvious influences on the recovery performance of the SMA wire, which can lead to low recovery performance. In addition, the recovery performance cannot fully meet the design index requirements. To further study the influence of parameter uncertainty on the motion reliability of the SMA wire in unlocking, 10,000 sets of sample data with the coefficient of variation within the range are analyzed based on the CPSO-BR-BP neural network proxy model, and the response value under each parameter combination is 


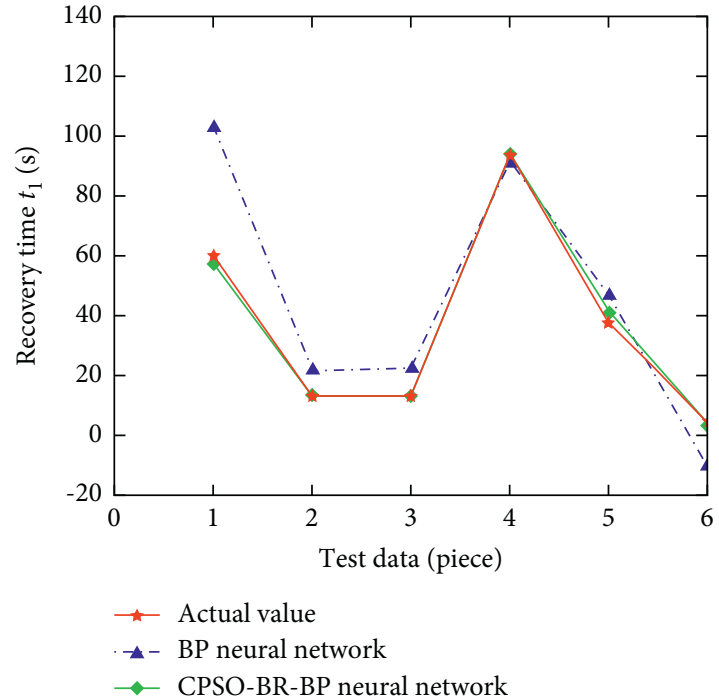

(a)

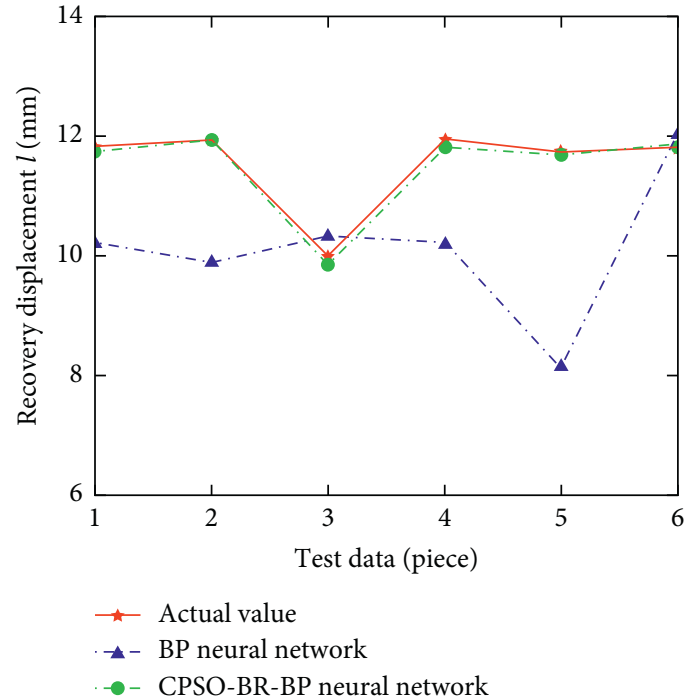

(b)

Figure 6: Comparison between predicted values and real values of surrogate model: (a) response time prediction and (b) recovery displacement prediction.

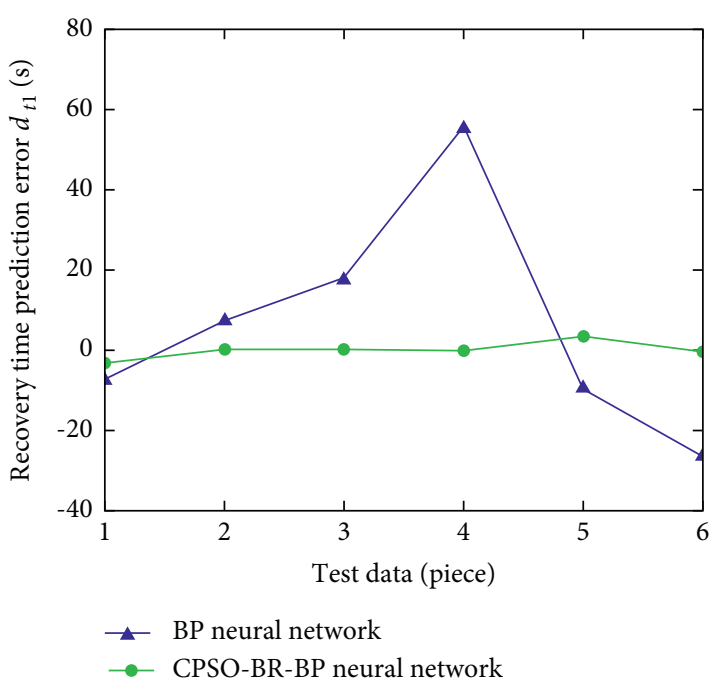

(a)

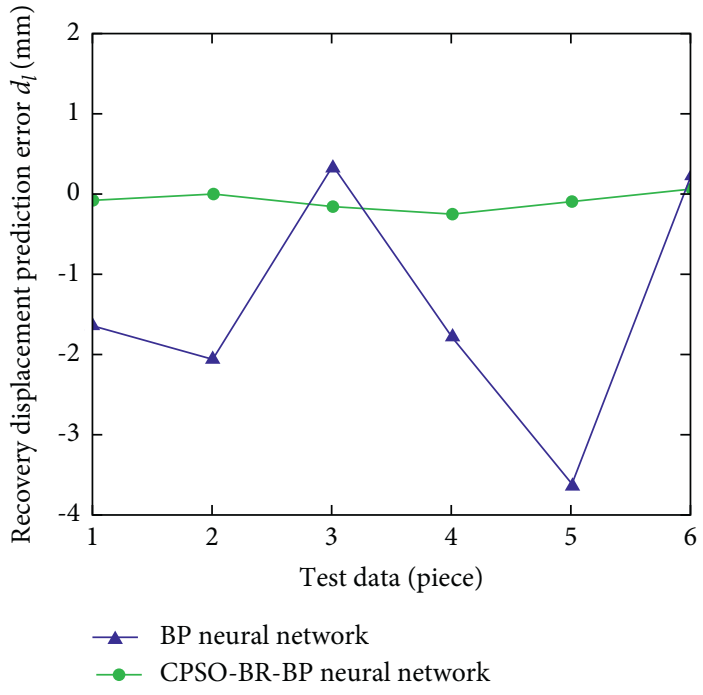

(b)

FIgURE 7: Prediction error of surrogate model: (a) recovery time and (b) recovery displacement.

TABLE 4: Prediction data of SMA wire under uncertainty parameter fluctuation.

\begin{tabular}{lccccccc}
\hline No. & Heating current & Heating time & Wire diameter & Load & Temperature & Response time & Response displacement \\
\hline 1 & 6.07 & 92.11 & 0.20 & 6.51 & 6.06 & 27.87 & 12.02 \\
2 & 5.21 & 105.89 & 0.30 & 8.49 & -34.89 & 30.43 & 11.79 \\
3 & 4.88 & 101.61 & 0.35 & 4.45 & 35.58 & 61.56 & $\ldots .47$ \\
$\ldots$ & $\ldots$ & $\ldots$ & $\ldots$ & $\ldots$ & $\ldots$ & $\ldots$ & $\ldots$ \\
9998 & 4.15 & 116.92 & 0.30 & 11.05 & -56.16 & 29.38 & 12.14 \\
9999 & 3.61 & 95.80 & 0.31 & 7.78 & 10.71 & 25.85 & 11.62 \\
10000 & 5.82 & 64.67 & 0.24 & 12.38 & -9.48 & 23.51 & 12.13 \\
\hline
\end{tabular}

predicted. The mean value and standard deviation of the recovery displacement of SMA wires with different coefficients of variation are calculated as shown in Figure 10.
It can be seen from Figure 10 that as the coefficient of variation increases, the standard deviation of the recovery displacement gradually increases, indicating that the 


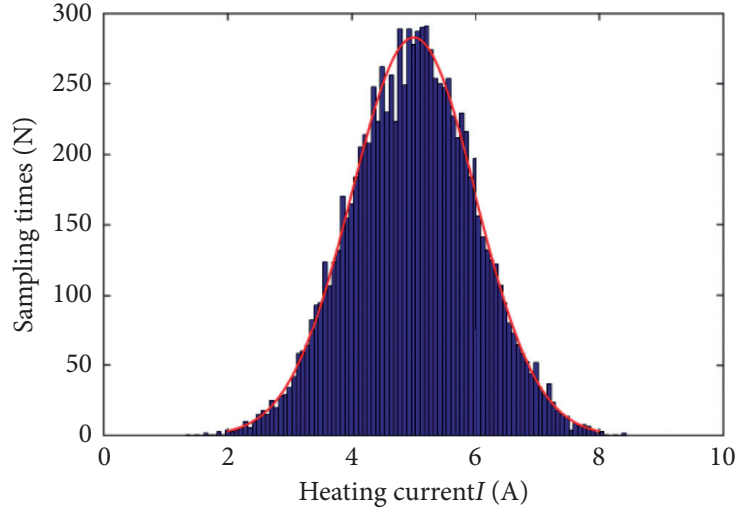

(a)

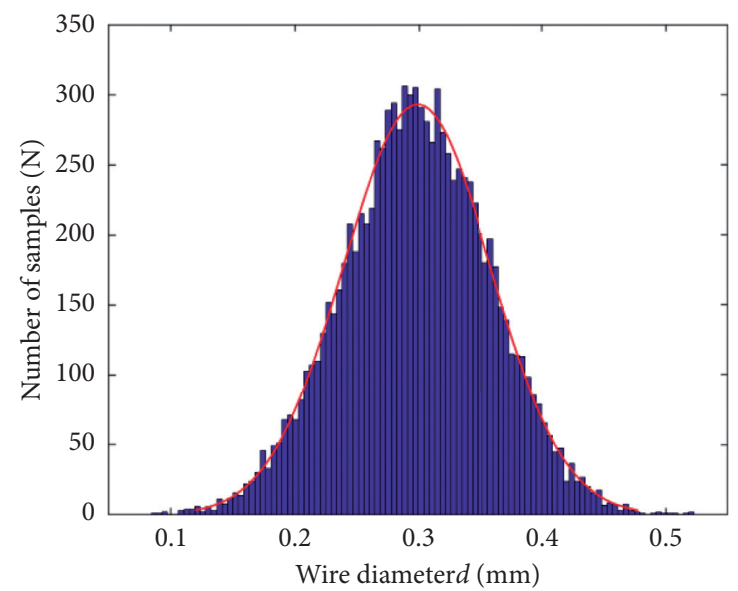

(c)

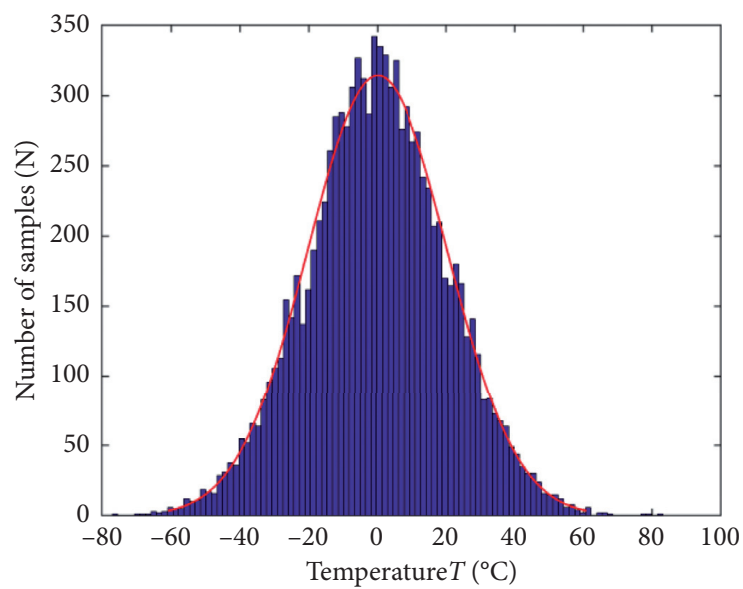

(e)

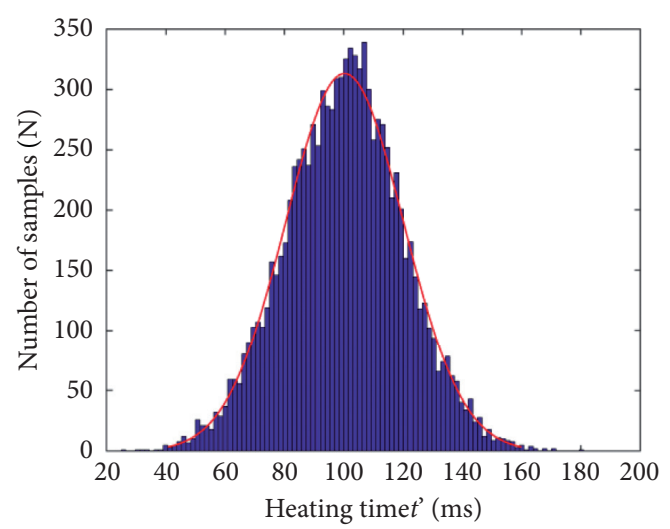

(b)

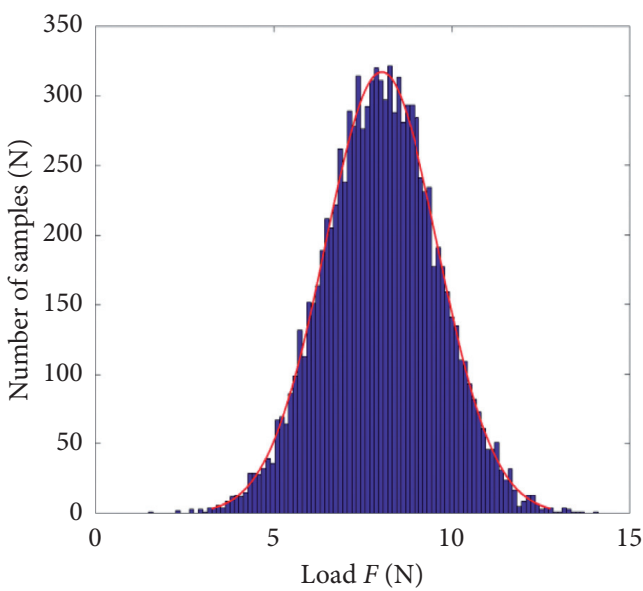

(d)

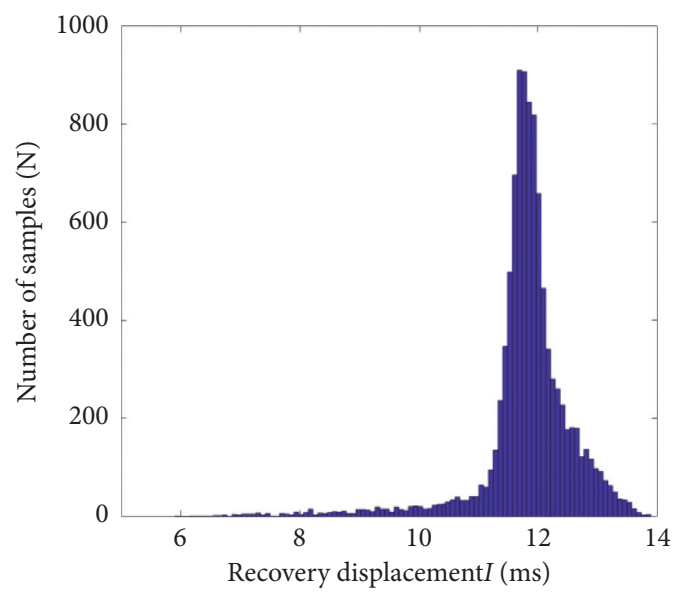

(f)

Figure 8: Probability distribution of heating current (a) $(\mathrm{N}(5,0.252))$, heating time (b) $(\mathrm{N}(100,52))$, wire diameter $(\mathrm{c})(\mathrm{N}(0.3,0.0152))$, load $(\mathrm{d})(\mathrm{N}(8,0.42))$, temperature $(\mathrm{e})(\mathrm{N}(0,0.42))$, and response displacement $(\mathrm{f})$.

increase in the coefficient of variation makes the dispersion of the uncertainty parameter values increase. The dispersion of the recovery displacement of the SMA wire becomes larger, which increases the possibility that the recovery displacement is smaller than the prescribed threshold. Secondly, the mean value of the return displacement of the SMA wire continues to decrease, indicating that the increase of the coefficient of variation makes the coupling effects of the uncertain parameters lead to the reduction of the recovery performance of the SMA wire. To further quantify the relationship between parameter uncertainty and SMA wire movement reliability, the movement reliability of the SMA wire in unlocking under different coefficients of variation is calculated, as shown in Figure 11. 


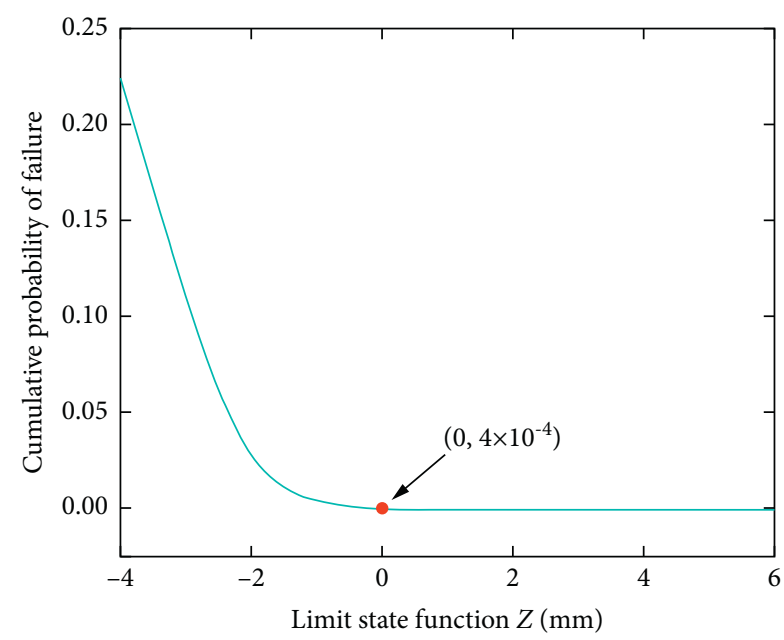

FIgURE 9: Cumulative failure probability of limit state function.

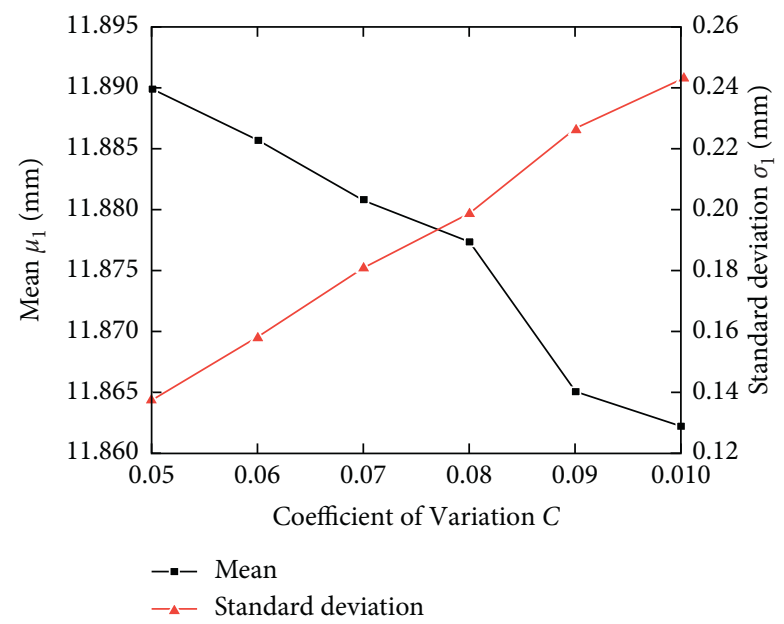

FIGURE 10: Mean and standard deviation of recovery displacement of SMA wires with different coefficients of variation.

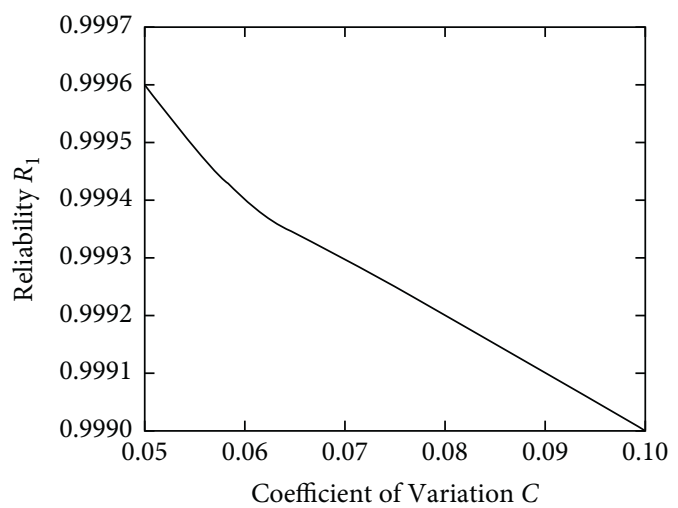

Figure 11: Kinematic reliability of SMA wire under different coefficients of variation.

It can be seen from Figure 11 that the movement reliability of the SMA wire in unlocking is continuously reduced with the increase of the uncertainty parameter variation coefficient. When the coefficient of variation is 0.05 , the highest working reliability of the device is 0.9996 . When the coefficient of variation is 0.10 , the lowest working reliability of the device is 0.999 , indicating that the parameter uncertainty has a significant impact on the movement reliability of the SMA wire. In work, the fluctuation range of various parameters should be strictly controlled to ensure the motion reliability of the SMA wire.

\subsection{Motion Reliability Analysis of Separation Process}

\subsubsection{Deterministic Dynamic Simulation of the Separation} Process. To consider the accuracy and efficiency of the motion reliability analysis of the separation process, ADAMS is used to simulate and analyze the motion process of the separation process. The deterministic analysis of the separation process is the basis and prerequisite to ensure the parameterization of the mechanism. For this reason, according to the simplified separation device, the virtual prototype model is established by ADAMS, as shown in Figure 12.

To accurately simulate the actual working conditions of the separation device, a measurement function of the distance between the top of the bearing screw and the bottom of the flywheel nut needs to be established. The simulation time is set to $0.1 \mathrm{~s}$ and the step length is 1000 steps. When the distance between the two is 0 , it means that the bearing screw completely withdraws from the thread pair; that is, the device separation ends, and the simulation stops. The calculation result is shown in Figure 13.

It can be seen from Figure 13 that the constraints of the separation device at all levels are released at $0 \mathrm{~s}$ (initial state), and the flywheel nut starts to release the stored pretightening force and drive the bearing screw to rotate at high speed. At this time, there is a gap between the thread pair, the flywheel nut, and the thrust roller bearing, so that the normal pressure between the contact surfaces is small. In addition, the friction resistance torque of the bearing screw is smaller, and the resultant torque is relatively stable, so the acceleration of the bearing screw remains unchanged from 0 to $0.0049 \mathrm{~s}$. As the separation process continues, the remaining pretightening force of the flywheel nut gradually decreases, and contact between the contact surfaces gradually occurs. At the same time, the normal pressure increases, and the friction resistance torque acting on the device continues to increase. In addition, the resultant torque continues to decrease, and the acceleration of the bearing screw gradually decreases within 0.0049-0.0184 s. During the entire movement, the speed of the bearing screw keeps increasing. When the separation process reaches $0.0184 \mathrm{~s}$, the remaining pretightening force of the flywheel nut is relatively balanced with the frictional resistance torque received by the device, and the total torque received by the bearing screw is 0 . In addition, the acceleration becomes 0 , and the bearing screw moves at a constant speed until $0.0604 \mathrm{~s}$ and completely exits the thread pair, completing the device separation process.

4.2.2. Analysis of the Influence of Parameter Uncertainty on Separation Time. According to the unlocking mechanism of the separation device and the ADAMS simulation results, it 


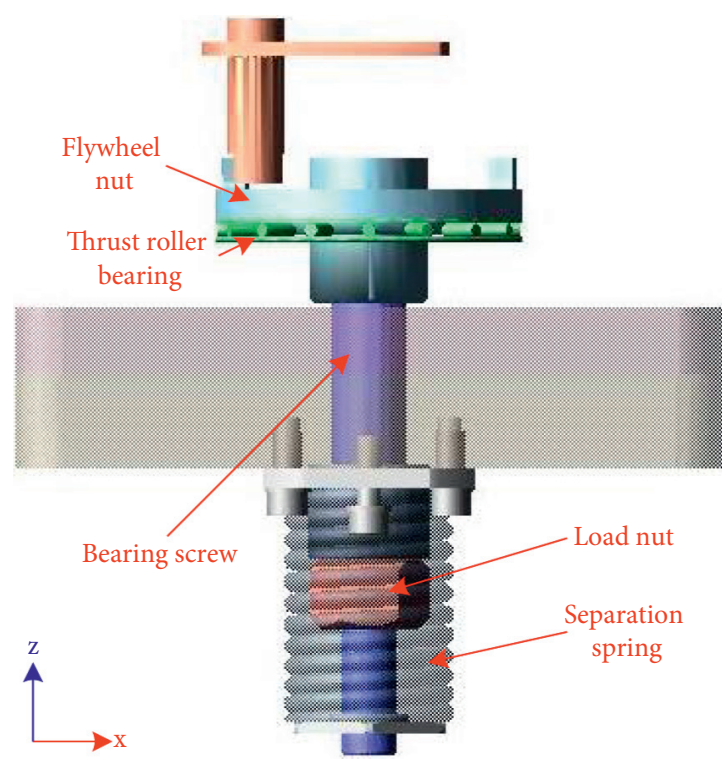

(a)

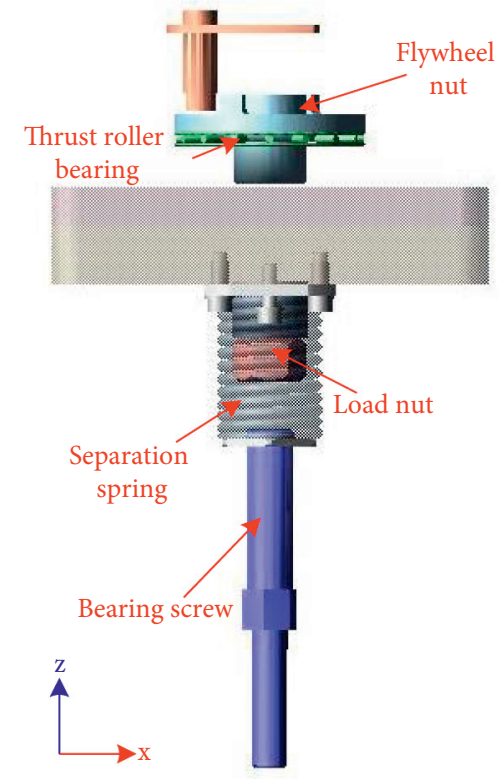

(b)

FIgURE 12: Virtual prototype model of separation device: (a) locking state and (b) unlocking state.

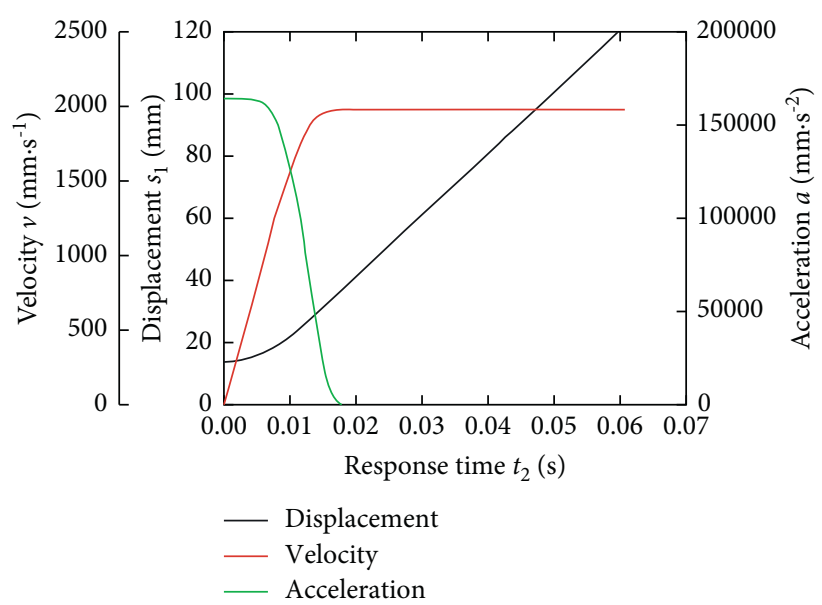

FIgURE 13: Simulation results of the separation process.

can be seen that the driving torque acting on the flywheel nut and the frictional resistance torque provided by the thrust roller bearing and the thread pair are the key factors affecting its kinematic parameters. Affected by various uncertain factors such as manufacturing, installation, and working environment, the force exerted on the separation device has certain randomness, which makes deviations from the theoretical value. Besides, this affects the separation time of the device and its working reliability. Therefore, three equal moments of driving torque, frictional resistance torque of thrust roller bearings, and frictional resistance torque of non-self-locking thread pairs are used as the uncertainty parameters that affect the reliability of the separation process. It is assumed that each parameter obeys the normal distribution with a coefficient of variation of 0.05 [24], and the characteristic values of its probability distribution are shown in Table 5.
The parametric virtual prototype model of the separation device is established based on the data provided in Table 5. 200 sets of samples are randomly selected by ADAMS for the three uncertainty parameters, and the motion simulation of the separation process is performed to obtain the uncertainty parameter combination under random sampling and its response value, as shown in Table 6 . The random value process of the uncertainty parameter and the changing trend of the separation time under different parameter combinations are shown in Figure 14.

It can be seen from Figure 14(a) that the sample values of the 200 sets of uncertainty parameters obtained by ADAMS simulation have good randomness and can more accurately characterize the changes of parameters caused by the uncertainty factors, such as driving torque, frictional resistance torque of thrust roller bearings, and frictional resistance 
TABLe 5: Probability distribution characteristics of uncertain parameters.

\begin{tabular}{lcccrc}
\hline Uncertainty parameter & Symbol & Unit & Mean & Coefficient of variation & Distribution type \\
\hline Driving torque & $T_{D}$ & $\mathrm{~N} \cdot \mathrm{mm}$ & 786 & 0.05 & Normal distribution \\
Friction torque of thrust roller bearings & $T_{m t b}$ & $\mathrm{~N} \cdot \mathrm{mm}$ & 229 & 0.05 & Normal distribution \\
Friction torque of thread pairs & $T_{m f}$ & $\mathrm{~N} \cdot \mathrm{mm}$ & 245 & 0.05 & Normal distribution \\
\hline
\end{tabular}

TABLE 6: Uncertain parameters and response values.

\begin{tabular}{lcccc}
\hline No. & Driving torque & Friction torque of thrust roller bearings & Friction torque of thread pairs & Separation time \\
\hline 1 & 731.434 & 246.719 & 268.045 & 0.0725 \\
2 & 739.074 & 226.417 & 238.174 & 0.0644 \\
3 & 808.139 & 234.354 & 244.573 & $\ldots .0588$ \\
$\ldots$ & $\ldots$ & $\ldots$ & 229.945 & $\ldots$ \\
198 & 835.707 & 223.411 & 270.760 & 0.0545 \\
199 & 807.476 & 220.945 & 271.858 & 0.0600 \\
200 & 884.286 & 218.235 & & 0.0537 \\
\hline
\end{tabular}

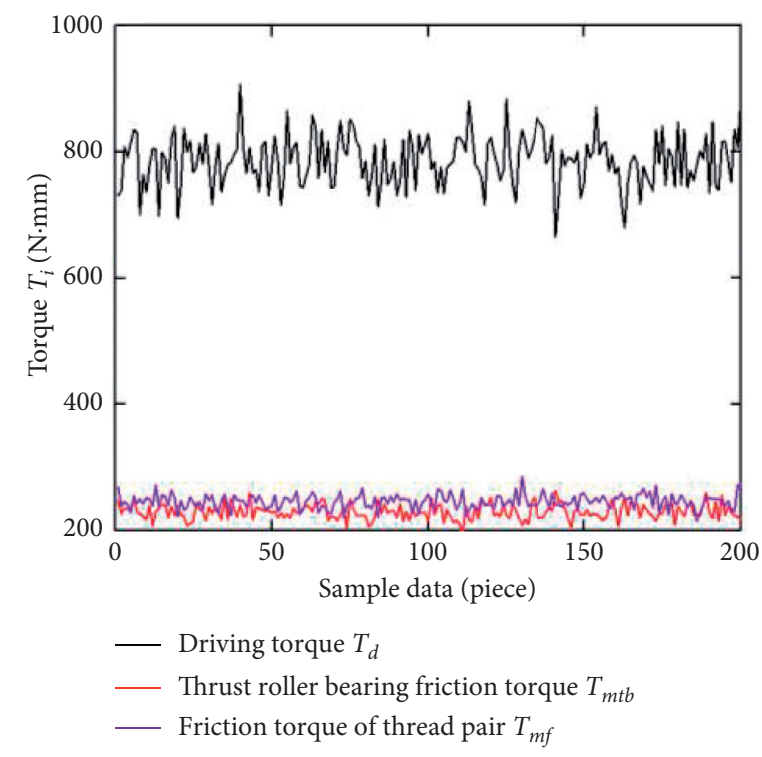

(a)

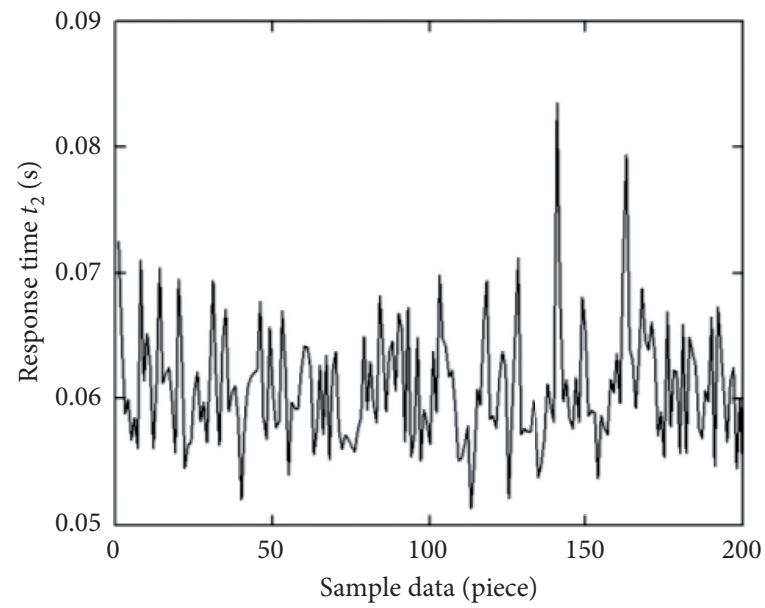

(b)

Figure 14: Sample value distribution of random variables (a) and response time (b).

torque of non-self-locking thread pairs. Figure 14(b) shows that, under the random fluctuation of parameters, the combination of parameters has a great impact on the separation time, and there are a few cases where the separation time exceeds the specified threshold. This indicates that the uncertainty of the parameters affects the movement reliability of the device's separation process. To more intuitively express the influence of the uncertainty parameters on the separation time, any two moments are selected as $X$-axis and $Y$-axis, and the sample space of different moments with respect to the separation time is drawn, as shown in Figure 15.

It can be seen from Figure 15 that the separation time of the device under the actions of any two moments shows a certain degree of dispersion, which is consistent with the random value of the parameter, and the phenomenon of clustering occurs in the sample space. This indicates that the random value range of the uncertainty parameter is relatively small, in line with the parameter fluctuation caused by uncertain factors under the conditions of continuously improving the processing and manufacturing level and the robust design. Secondly, most of the separation time is concentrated between 0.06 and $0.07 \mathrm{~s}$, and a small part exceeds the specified threshold, which shows that the uncertainty of the moment leads to the reduction of the reliability of the separation process.

The motion reliability analysis of the separation process should be performed based on a large number of samples. However, more accurately reliable results cannot be obtained based on the sample points provided in Table 6. In addition, the efficiency to determine the sample data using 


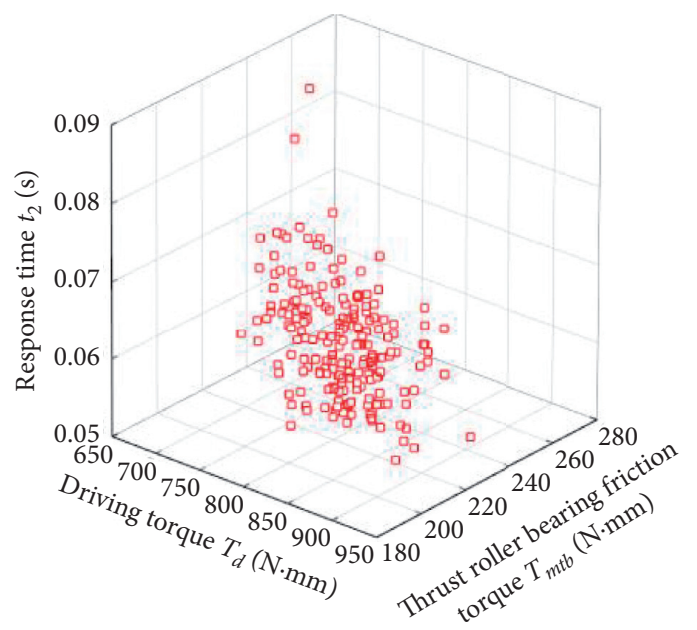

(a)

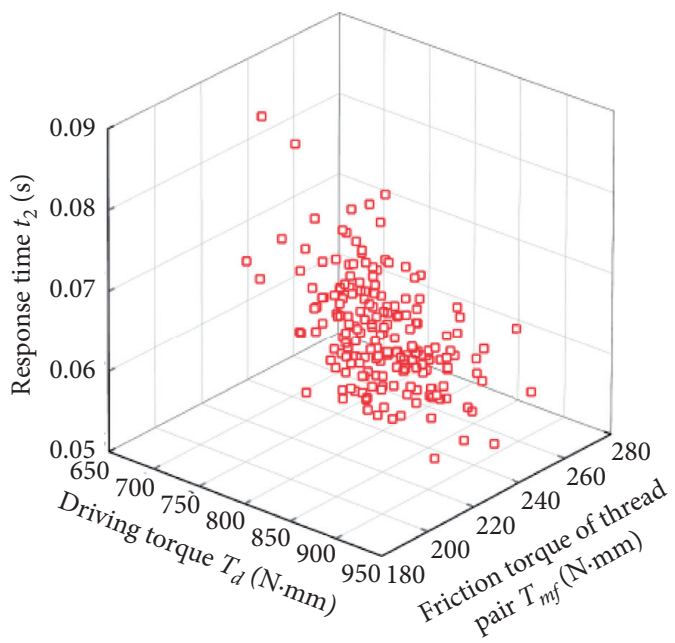

(b)

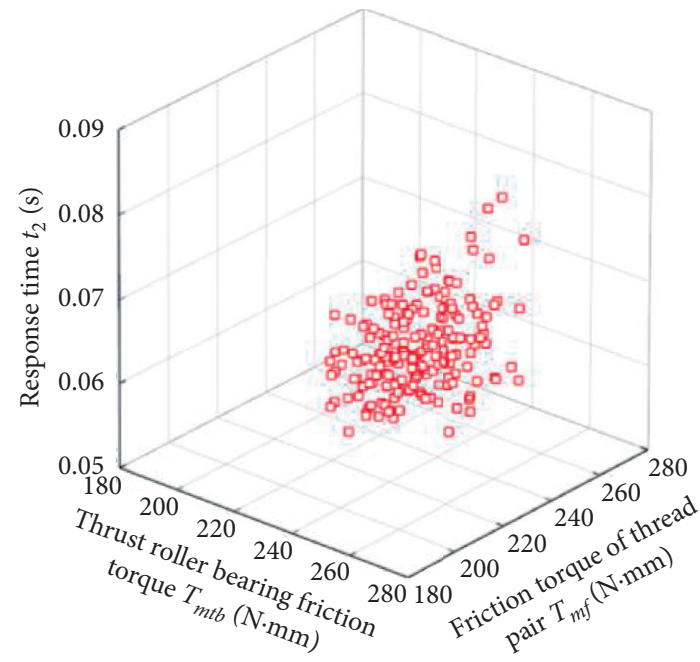

(c)

FIGURE 15: Sample space of uncertain parameters about separation time: (a) driving torque and friction torque of thrust roller bearings, (b) driving torque and friction torque of thread pairs, and (c) friction torque of thrust roller bearings and friction torque of thread pairs.

ADAMS simulation is low. For this reason, the proposed CPSO-BR-BP neural network proxy model is used to characterize the mapping relationship between the three moments and the separation time to improve the efficiency of motion reliability analysis.

170 sets of training samples are selected randomly as listed in Table 6 and normalized. According to the proposed method, the proxy model of three moments for separation time is established, and the expression is

$$
\begin{aligned}
t_{2}= & -\frac{0.3220}{C_{1}}+\frac{0.6547}{C_{2}}+\frac{0.6537}{C_{3}}+\frac{0.6744}{C_{4}} \\
& +\frac{1.1736}{C_{5}}-\frac{2.4037}{C_{6}}+\frac{0.9187}{C_{7}}+0.4896,
\end{aligned}
$$

where

$$
\begin{aligned}
& C_{1}=1+\exp \left(0.3005 T_{D}-0.2550 T_{m t b}+0.4176 T_{m f}-0.0161\right), \\
& C_{2}=1+\exp \left(-0.3511 T_{D}+0.0938 T_{m t b}+0.1603 T_{m f}+0.0533\right), \\
& C_{3}=1+\exp \left(-0.3548 T_{D}+0.0944 T_{m t b}+0.1475 T_{m f}+0.0524\right), \\
& C_{4}=1+\exp \left(-0.3277 T_{D}+0.0934 T_{m t b}+0.2552 T_{m f}+0.0769\right), \\
& C_{5}=1+\exp \left(-0.9894 T_{D}+0.1032 T_{m t b}+0.2814 T_{m f}-1.3137\right), \\
& C_{6}=1+\exp \left(2.3090 T_{D}-0.7647 T_{m t b}-0.6694 T_{m f}+3.8676\right), \\
& C_{7}=1+\exp \left(-1.1269 T_{D}+0.3873 T_{m t b}+0.3199 T_{m f}-0.5233\right) .
\end{aligned}
$$

The remaining 30 sets of sample data in Table 6 are used as test samples to test the accuracy of model fitting. The sample data is brought into the proxy model to compare and analyze the actual value and predicted value, and the 
coefficient of determination is 0.99994 . The comparative analysis result is shown in Figure 16.

It can be seen from Figure 16 that the predicted value based on the CPSO-BR-BP neural network proxy model is consistent with the actual value change trend, which shows that the model can more accurately represent the mapping relationship. To further characterize the fitting accuracy of the surrogate model, the error distribution is shown in Figure 17. It can be seen from Figure 17 that although the prediction errors of the 30 sets of data fluctuate to varying degrees, their magnitudes are $10^{-5}$, indicating that the established proxy model has high accuracy and can accurately predict the separation time of the device under different moment values.

4.2.3. Motion Reliability Analysis of Separation Process. Based on the high-precision CPSO-BR-BP neural network proxy model, 10,000 Monte Carlo simulations are performed on the uncertain parameters, and the separation time of the device under each set of parameter combinations is predicted, as shown in Table 7. The random value of each parameter and the probability distribution characteristics of the corresponding separation time are shown in Figure 18.

It can be seen from Figures 18(a)-18(c) that the random distribution of each uncertainty parameter follows the normal distribution, which is basically consistent with the fluctuation form of the real situation, and the separation time of the device can also be predicted. The time for the device to complete the separation process under the uncertainty of parameter fluctuations is shown in Figure 18(d). Overall, the graph is affected by the parameter fluctuations, which make the separation time discrete, and it approximately obeys the probability characteristics of the normal distribution. However, there are a few cases where the response value exceeds the specified threshold at the censoring point, indicating that the uncertainty of the parameters affects the reliability of the separation process to a certain extent.

Incorporating the predicted separation time into equations (9)-(12), the motion reliability of the device separation process under the improved membership function is obtained. The undetermined coefficient $d$ directly affects the profile of the membership function. As the value of $d$ increases, the improved membership function gradually tends to the traditional rectangular membership function. To verify the advantages of the improved membership function, it is compared with the traditional rectangular membership function and the Monte Carlo simulation analysis results, as shown in Figure 19.

It can be seen from Figure 19 that the motion reliability obtained based on the improved membership function gradually tends to the Monte Carlo result as the number of iterations of the undetermined coefficient $d$ increases. When the number of iterations of $d$ reaches 25 times, the improved membership function is 0.9991 , and the motion reliability calculated by the Monte Carlo method is 0.9998 . The error between them is $0.07 \%$. However, the motion reliability of the separation process based on the traditional rectangular

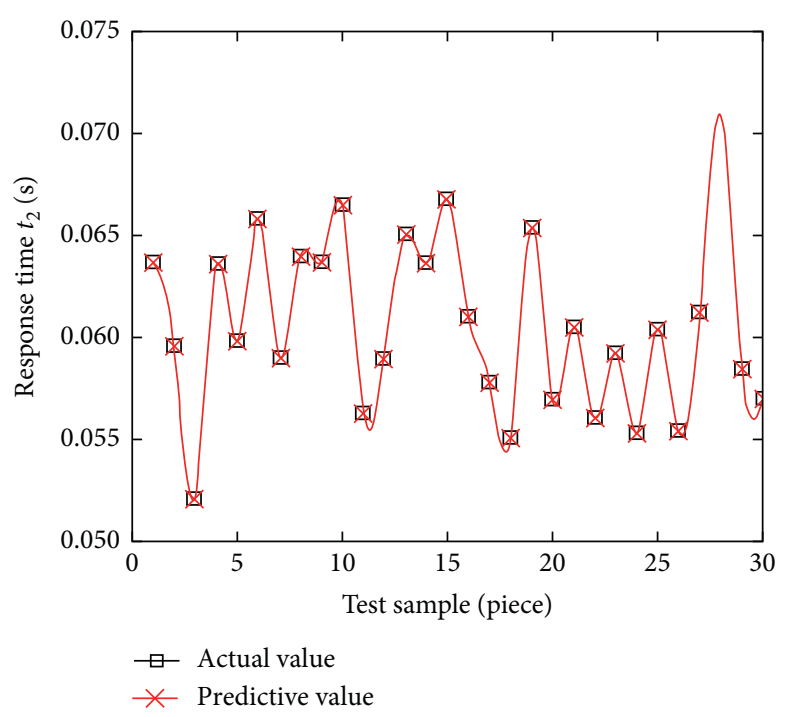

Figure 16: Comparison of predicted and actual values.

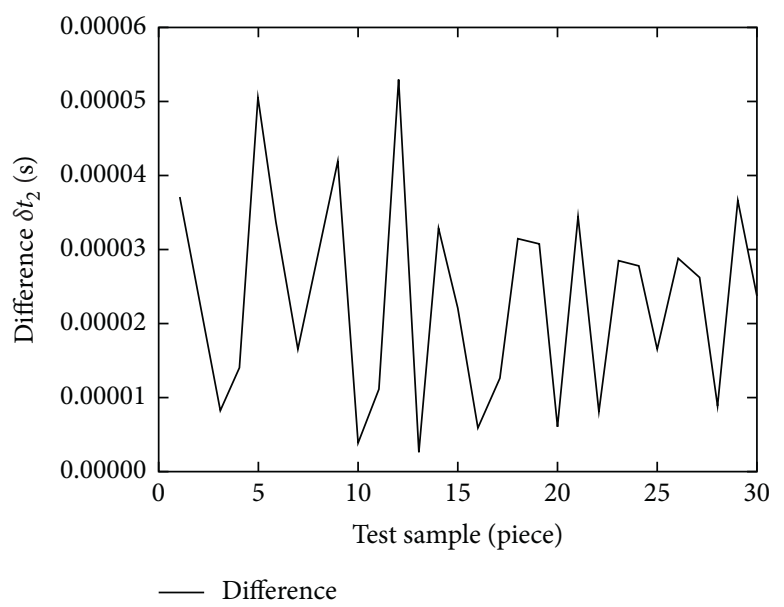

Figure 17: Data prediction error of 30 groups.

membership function is not affected by the undetermined coefficient $d$, and the result is always maintained at 0.9763 , the error of which is $2.35 \%$ compared with the Monte Carlo method. The comparative analysis shows that the use of the improved membership function to process the uncertainty parameters can better reflect the actual motion reliability of the device. The reliability calculation method based on the improved membership function is also certified to be of high accuracy.

To further study the influence of the uncertainty of the torque on the motion reliability of the separation process, the CPSO-BR-BP neural network proxy model is used to generate 10,000 sets of sample data with a coefficient of variation of 0.05-0.10 and to predict the response value of each parameter combination. The mean and standard deviation of the separation time under different coefficients of variation are obtained, as shown in Figure 20.

It can be seen from Figure 20 that both the mean and the standard deviation of separation time are positively correlated with the moment variation coefficient. This indicates 
TABLE 7: Forecast data when the coefficient of variation is 0.05 .

\begin{tabular}{lcccc}
\hline No. & Driving torque & Friction torque of thrust roller bearings & Friction torque of thread pairs & Separation time \\
\hline 1 & 801.4149 & 230.8262 & 249.5258 & 0.059537 \\
2 & 830.3701 & 243.9656 & 236.3860 & 0.056992 \\
3 & 709.8324 & 228.5464 & 222.1646 & $\ldots .066253$ \\
$\ldots$ & $\ldots$ & $\ldots$ & 245.1185 & $\ldots$ \\
9998 & 772.9308 & 242.3348 & 239.0925 & 0.063150 \\
9999 & 798.3322 & 205.5270 & 248.8006 & 0.056708 \\
10000 & 827.3862 & 227.1700 & & 0.056889 \\
\hline
\end{tabular}

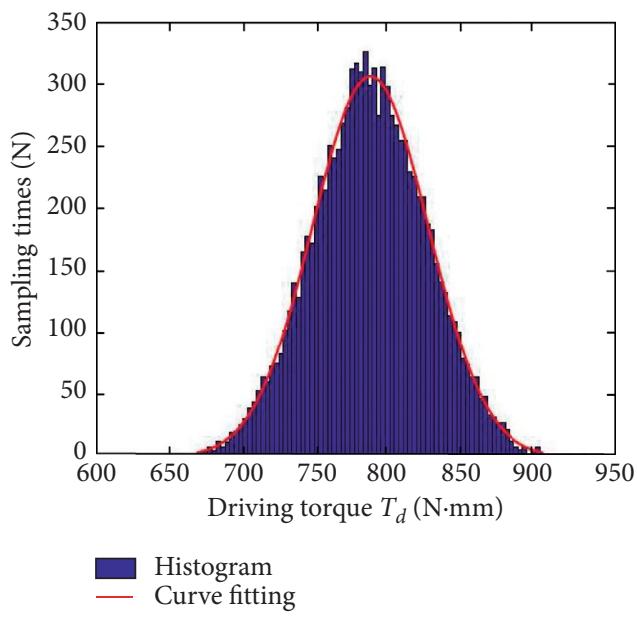

(a)

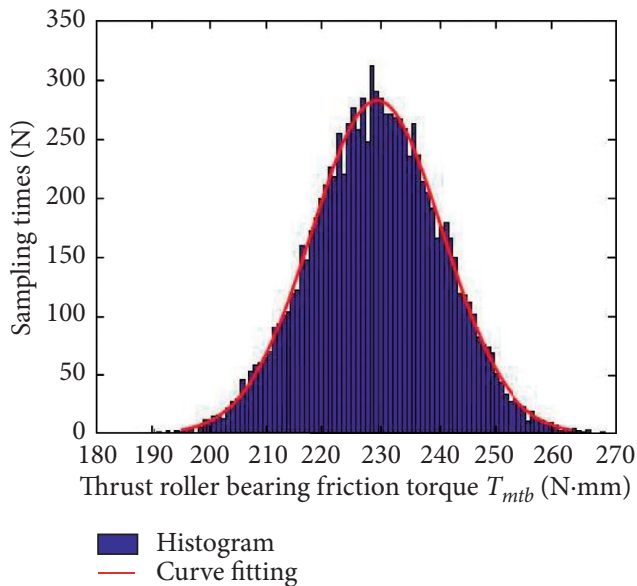

(c)

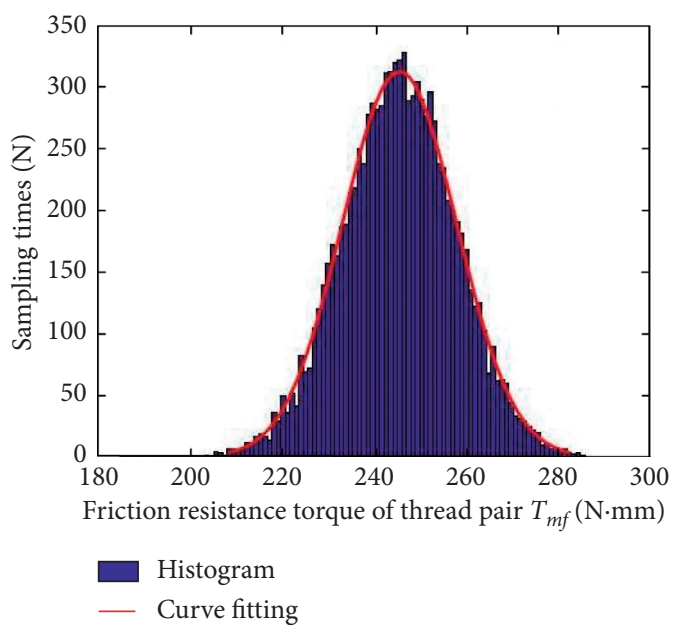

(b)

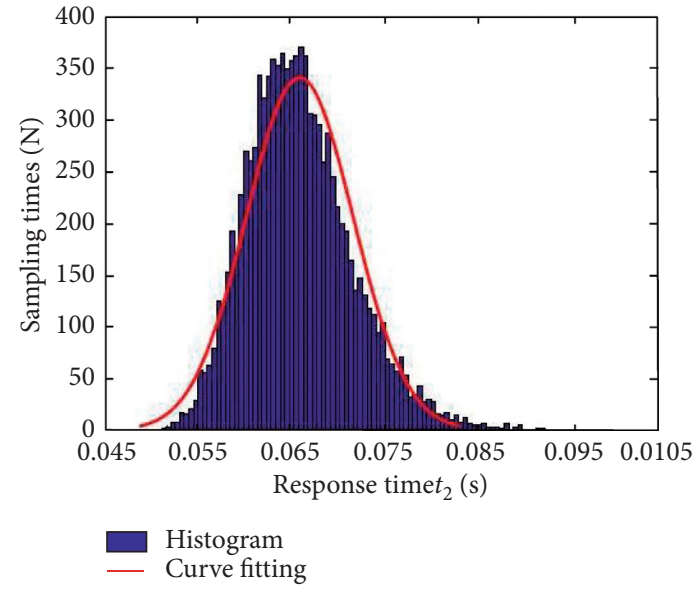

(d)

Figure 18: Probability distribution characteristics of driving torque (a) $(\mathrm{N}(786,39.32))$, friction torque of thrust roller bearings (b) ( $\mathrm{N}(229$, $11.452))$, friction torque of thread pairs (c) $(\mathrm{N}(245,12.252))$, and response time (d) $(\mathrm{N}(0.0607,0.00432))$.

that as the moment variation coefficient increases, the uncertainty of the resultant moment acting on the load-bearing screw gradually increases, which expands the separation time fluctuation range. As a result, the standard deviation of the separation time continues to increase, and the possibility of exceeding the threshold becomes great, which affects the reliability of the separation process of the device to a certain extent. To quantify the relationship between the moment uncertainty and the movement reliability of the device separation process, the separation time obtained in Figure 20 is substituted into equations (9)-(12) to obtain the movement reliability of the device separation process under different coefficients of variation, as shown in Figure 21.

It can be seen from Figure 21 that the motion reliability of the separation process is negatively correlated with the moment variation coefficient, and the changing trend of the motion reliability obtained based on the improved membership function is consistent with the Monte Carlo method. The value of the result is smaller than that of the Monte Carlo method because of the fuzzy treatment of uncertainty of 


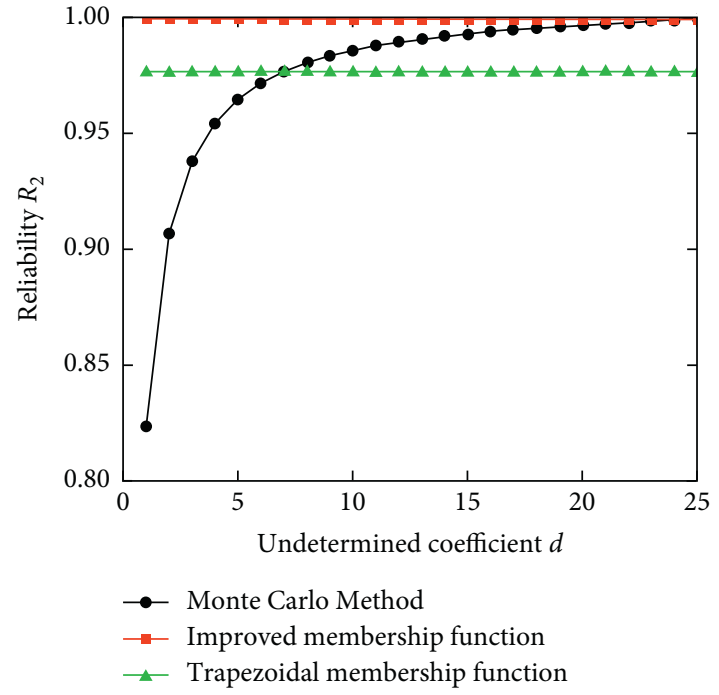

FIGURE 19: The iterative process of motion reliability for the separation process.

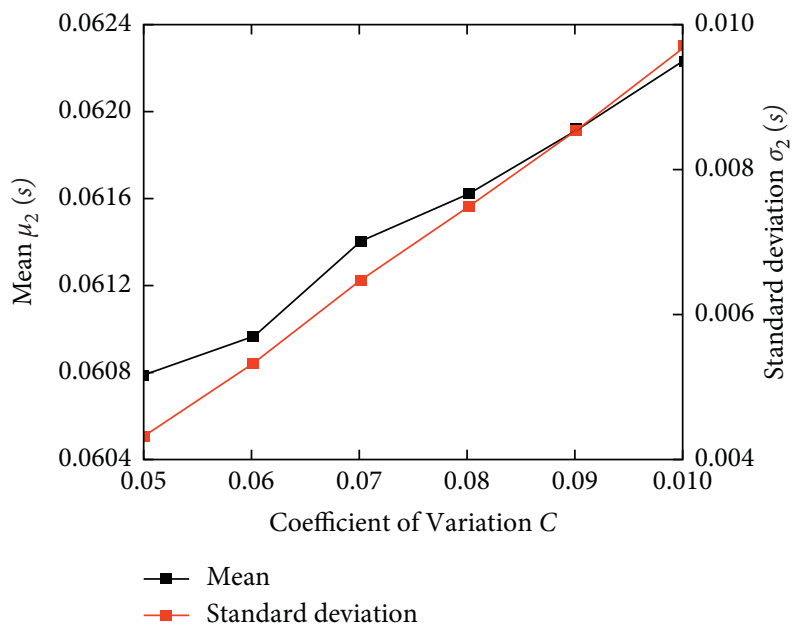

FIGURe 20: Mean and standard deviation of separation time under different coefficients of variation.

moment in the reliability calculation process. When the traditional rectangular membership function is used for calculation, the variation range of uncertainty parameters is large. Such variation range cannot reflect the manufacturing requirements of the actual device, resulting in a large error in the calculation compared with the Monte Carlo method.

The comparative analysis shows that the movement reliability of the device separation process under different coefficients of variation can be accurately obtained based on the improved membership function. When the coefficient of variation is 0.05 , the maximum reliability of the separation process is 0.9991 , and when the coefficient of variation is 0.10 , the minimum movement reliability of the separation process is 0.9429 , indicating that the moment uncertainty has a great influence on the movement reliability of the separation process. To ensure the working reliability of the separation device, the size of each moment should be strictly controlled, and the dispersion degree of each moment

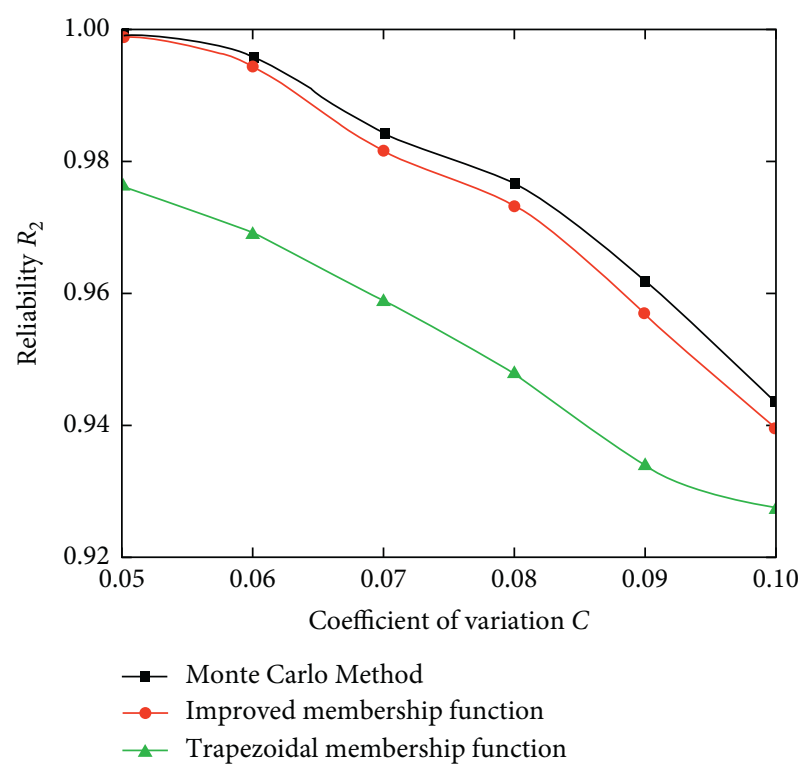

FIGURE 21: Motion reliability of separation process under different coefficients of variation.

should be reduced in the actual application of unlocking the trigger device.

4.3. Motion Reliability Analysis of Unlocking Trigger Device. According to the working principle of the unlocking trigger device, the reliability of the whole machine is composed of two parts of the reliabilities of the SMA wire unlocking and the separation process. To accurately characterize the influence of parameter uncertainty on the reliability of the whole machine, the reliability of the whole machine is calculated based on the established device reliability model, and the expression is

$$
R_{t}=R_{1} \times R_{2}=0.9996 \times 0.9991=0.9987,
$$

where $R_{1}$ is the motion reliability of the SMA wire in unlocking and $R_{2}$ is the motion reliability of the separation process.

It can be seen from equation (21) that the reliability of the unlocking trigger device is 0.9987 , which is lower than the reliability requirement of the design index 0.9999 . The reason is that the influence of parameter uncertainty on the reliability of each part is considered in the analysis process. The reliability of the whole machine is lower than the reliability of each part under the separate action, indicating that the reliability of each part of the device has a great impact on the reliability of the whole machine. In the design process, it is necessary to strictly control the reliability of the SMA wire unlocking movement and the separation process to improve the reliability of the whole machine. To further explore the relationship between the parameter uncertainty and the overall reliability of the device, the series reliability model is used to calculate the reliability of the device under different coefficients of variation. The analysis result is shown in Figure 22. 


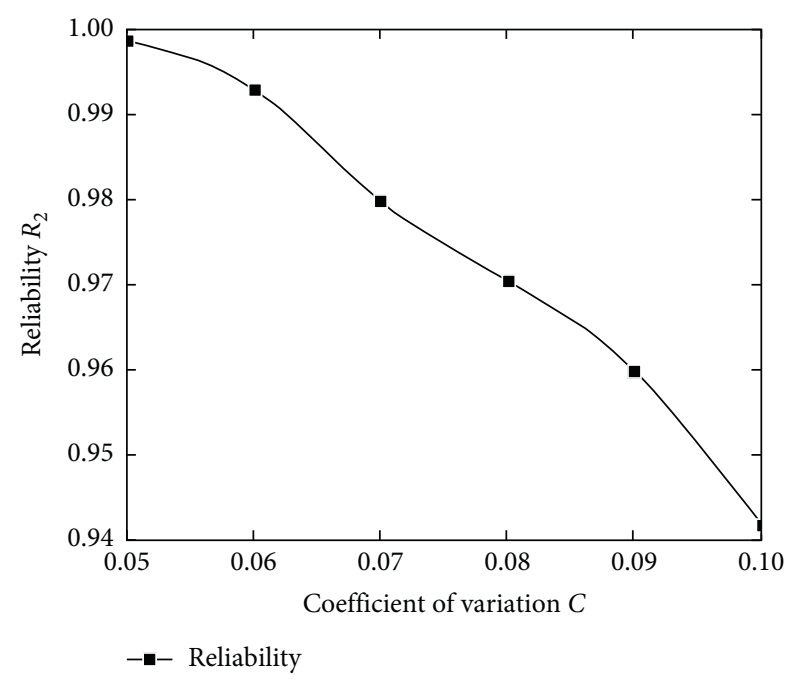

Figure 22: Reliability of the whole machine under different coefficients of variation.

It can be seen from Figure 22 that as the coefficient of variation of the uncertainty parameter increases, the reliability of the whole machine gradually decreases. When the coefficient of variation is 0.05 , the reliability of the device is 0.9987 ; when the coefficient of variation is 0.10 , the reliability of the device is 0.9419 . The greater the uncertainty of the parameters is, the less the probability that the overall performance of the device meets the design index, which reduces the reliability of the device. To ensure that the device can work with high reliability, the influence of uncertain factors should be reduced as much as possible.

\section{Conclusions}

(1) Considering the influence of parameter uncertainty on the motion reliability of the unlocking trigger device, the BP neural network is optimized by combining CPSO and BR. Based on the construction of the unlocking trigger device reliability model, the CPSO-BR-BP neural network agent is established. Compared with the traditional neural network proxy model, the proposed proxy model has higher fitting accuracy. It can better characterize the mapping relationship between inputs and outputs and improve the efficiency of reliability calculation. Furthermore, it can save calculation costs.

(2) Based on the CPSO-BR-BP neural network proxy model, it is calculated that the motion reliability of the SMA wire in unlocking is 0.9996 , the movement reliability of the separation process is 0.9991 , and the movement reliability of the whole machine is 0.9987 , which are under the condition of parameter uncertainty. The uncertainty of the parameters in the process of SMA wire in the unlocking and separation process makes the reliability of the whole machine lower than the reliability design index, but it is larger in line with the real reliability of the unlocking trigger device.
(3) The influences of different coefficients of variation on the motion reliability of SMA wire unlocking, the separation process, and the whole machine are compared and analyzed. The motion reliabilities of the three cases all show different degrees of reduction with the increase of the coefficient of variation, indicating that the degree of dispersion of parameters has a great impact on the dispersion of the response. Ultimately, it can result in the reduction of the motion reliability of the whole machine. Therefore, to ensure the stability of the device unlocking and separation process, the influences of uncertain factors should be minimized.

\section{Data Availability}

No data were used to support this study.

\section{Conflicts of Interest}

The authors declare that they have no conflicts of interest.

\section{References}

[1] M. Yazdi, S. Daneshvar, and H. Setareh, "An extension to fuzzy developed failure mode and effects analysis (FDFMEA) application for aircraft landing system," Safety Science, vol. 98, pp. 113-123, 2017.

[2] C.-G. Huang, H.-Z. Huang, and Y.-F. Li, "A bidirectional LSTM prognostics method under multiple operational conditions," IEEE Transactions on Industrial Electronics, vol. 66, no. 11, pp. 8792-8802, 2019.

[3] M. Yazdi and E. Zarei, "Uncertainty handling in the safety risk analysis: an integrated approach based on fuzzy fault tree analysis," Journal of Failure Analysis and Prevention, vol. 18, no. 2, pp. 392-404, 2018.

[4] W. Peng, Z.-S. Ye, and N. Chen, "Bayesian deep-learningbased health prognostics toward prognostics uncertainty," IEEE Transactions on Industrial Electronics, vol. 67, no. 3, pp. 2283-2293, 2020.

[5] M. Yazdi, S. Kabir, and M. Walker, "Uncertainty handling in fault tree based risk assessment: state of the art and future perspectives," Process Safety and Environmental Protection, vol. 131, pp. 89-104, 2019.

[6] Z. Zhong, H. Zhang, J. Zhou et al., "Research review of spacecraft non-pyrotechnic connection and separation technology," Manned Spaceflight, vol. 25, no. 1, pp. 128-142, 2019.

[7] J. Zhou, “The overall concept of my country's space station project," Manned Spaceflight, vol. 19, no. 2, pp. 1-10, 2013.

[8] X. Zhang, X. Yan, and Q. Yang, "Design and experimental validation of compact, quick-response shape memory alloy separation device," Journal of Mechanical Design, vol. 136, pp. 1-9, 2014.

[9] X. Hu, J. Wu, J. Peng et al., "Design and experimental study of large-load low-impact unlocking mechanism based on memory alloy," Science Technology and Engineering, vol. 16, no. 30, pp. 319-323, 2016.

[10] M. Han, D. Wang, Y. Tian et al., "Design and analysis of micro-nano satellite linkage connection and separation device," Mechanical Science and Technology, vol. 39, no. 9, pp. 1463-1470, 2020. 
[11] L. Hong, H. Li, K. Peng et al., "Improved response surface method for reliability analysis based on efficient search method," Journal of Beijing University of Aeronautics and Astronautics, vol. 46, no. 1, pp. 95-102, 2020.

[12] P. Zhang, Z. Zhang, and H. Guo, "Optimal design of tapered hydrostatic bearing based on Kriging model," Modular Machine Tool and Automatic Manufacturing Technology, no. 2, pp. 62-65+68, 2021.

[13] Z.-H. Han, Y. Zhang, C.-X. Song, and K.-S. Zhang, "Weighted gradient-enhanced Kriging for high-dimensional surrogate modeling and design optimization," AIAA Journal, vol. 55, no. 12, pp. 4330-4346, 2017.

[14] Z. Wen, H. Pei, H. Liu, and Z. Yue, "A sequential Kriging reliability analysis method with characteristics of adaptive sampling regions and parallelizability," Reliability Engineering \& System Safety, vol. 153, pp. 170-179, 2016.

[15] J. Zhao, Q. C. Jian, and L. Xu, "RBF-GA: an adaptive radial basis function metamodeling with genetic algorithm for structural reliability analysis," Reliability Engineering \& System Safety, vol. 189, pp. 42-57, 2019.

[16] L. Shi, B. Sun, and D. S. Ibrahim, "An active learning reliability method with multiple kernel functions based on radial basis function," Structural and Multidisciplinary Optimization, vol. 60, no. 1, pp. 211-229, 2019.

[17] S. Chen and D. Wang, "Monte Carlo reliability analysis method based on neural network," Journal of Shanghai Jiaotong University, vol. 52, no. 6, pp. 687-692, 2018.

[18] A. Hraiba, A. Touil, and A. Mousrij, "Artificial neural network based hybrid metaheuristics for reliability analysis," IFACPapersOnline, vol. 53, no. 1, pp. 654-660, 2020.

[19] J. Izquierdo, A. Crespo Márquez, and J. Uribetxebarria, "Dynamic artificial neural network-based reliability considering operational context of assets," Reliability Engineering \& System Safety, vol. 188, pp. 483-493, 2019.

[20] C. Tang, J. Zhang, W. Hou et al., "Reliability sensitivity analysis of motion mechanism based on artificial neural network," Forging Technology, vol. 44, no. 8, pp. 182-188, 2019.

[21] C. Dai, W. Chen, and Y. Zhu, "Seeker optimization algorithm for tuning the structure and parameters of neural networks," Neurocomputing, vol. 74, no. 6, 2010.

[22] H. Gong, Z. Li, H. Liu et al., "Storage reliability prediction based on improved PSO-BP neural network," Operations Research and Management, vol. 29, no. 8, pp. 105-111, 2020.

[23] Y. Jin, S. Liu, and J. Zhang, "Fatigue reliability of high-speed rolling bearings under artificial neural network optimized by genetic algorithm," Journal of Aeronautics and Dynamics, vol. 33, no. 11, pp. 2748-2755, 2018.

[24] K. Sun, J. Zhou, and L. Xie, "Reliability optimization design of CNC machine tool ball screw," Mechanical Science and Technology, vol. 29, no. 11, pp. 1530-1533, 2010.

[25] P. Zhi, Y. Li, and B. Chen, "Structural strength analysis of bogie frame considering parameter uncertainty," China $\mathrm{Me}$ chanical Engineering, vol. 30, no. 1, pp. 22-29, 2019. 